\title{
Configurations, Power Topologies and Applications of Hybrid Distribution Transformers
}

\author{
Alvaro Carreno ${ }^{1,+}+\mathbb{D}$, Marcelo Perez ${ }^{1, *,+} \mathbb{D}$, Carlos Baier ${ }^{2,+} \mathbb{D}$, Alex Huang ${ }^{3,+}\left(\mathbb{D}\right.$, Sanjay Rajendran ${ }^{3,+} \mathbb{D}$ and \\ Mariusz Malinowski $4, *,+\mathbb{D}$
}

1 Electronics Engineering Department, Universidad Tecnica Federico Santa Maria, Valparaiso 2390123, Chile; alvaro.carreno@alumnos.usm.cl

2 Department of Electrical Engineering, Universidad de Talca, Curico 3340000, Chile; cbaier@utalca.cl

3 Semiconductor Power Electronics Center, The University of Texas, Austin, TX 78712, USA; aqhuang@utexas.edu (A.H.); sanjayrajendran@utexas.edu (S.R.)

4 Institute of Control and Industrial Electronics, Warsaw University of Technology, 00-662 Warsaw, Poland

* Correspondence: marcelo.perez@usm.cl (M.P.); malin@ee.pw.edu.pl (M.M.)

+ These authors contributed equally to this work.

check for updates

Citation: Carreno, A.; Perez, M.; Baier, C.; Huang, A.; Rajendran, S.; Malinowski, M. Configurations, Power Topologies and Applications of Hybrid Distribution Transformers. Energies 2021, 14, 1215. https:// doi.org/10.3390/en14051215

Academic Editor: Branislav Hredzak

Received: 2 January 2021

Accepted: 11 February 2021

Published: 24 February 2021

Publisher's Note: MDPI stays neutral with regard to jurisdictional claims in published maps and institutional affiliations.

Copyright: (c) 2021 by the authors. Licensee MDPI, Basel, Switzerland. This article is an open access article distributed under the terms and conditions of the Creative Commons Attribution (CC BY) license (https:/ / creativecommons.org/licenses/by/ $4.0 /)$.

\begin{abstract}
Distribution systems are under constant stress due to their highly variable operating conditions, which jeopardize distribution transformers and lines, degrading the end-user service. Due to transformer regulation, variable loads can generate voltage profiles out of the acceptable bands recommended by grid codes, affecting the quality of service. At the same time, nonlinear loads, such as diode bridge rectifiers without power factor correction systems, generate nonlinear currents that affect the distribution transformer operation, reducing its lifetime. Variable loads can be commonly found at domiciliary levels due to the random operation of home appliances, but recently also due to electric vehicle charging stations, where the distribution transformer can cyclically vary between no-load, rated and overrated load. Thus, the distribution transformer can not safely operate under highly-dynamic and stressful conditions, requiring the support of alternative systems. Among the existing solutions, hybrid transformers, which are composed of a conventional transformer and a power converter, are an interesting alternative to cope with several power quality problems. This article is a review of the available literature about hybrid distribution transformers.
\end{abstract}

Keywords: distribution transformers; hybrid transformers; partial power converter; power electronics converter; power quality; transformer lifetime

\section{Introduction}

Distribution transformers are one of the pillars of the distribution grid. They are the interface between the medium and low-voltage grid, stepping-down the voltages to a safe and usable range for the end-users. They are characterized by being a highly-robust and low-cost solution, being available in different winding configurations and power ratings according to the grid requirement and mounting type [1]. Transformers have an average lifetime above 35 years under rated conditions [2].

Power quality is a term employed when referring to the power system voltage and current waveform quality at different points of the grid [3]. Low power quality phenomenons can be generated by the users, for example when consuming nonsinusoidal and unbalanced currents. Weak grids can be a source of low power quality, providing unbalanced and variable frequency voltages, among others. IEEE Std 1159-2019 and IEEE Std 519-2014 give guides on power quality measurements and recommended practice to comply and guarantee a good quality service [4,5]. Some effects of low power quality are high voltage peaks that can damage equipment, heating, losses, acoustic noise, and lifetime reduction in equipment. For utilities, low power quality is translated into additional losses on transmission lines and generators or the failure of power systems elements that can 
jeopardize the correct grid operation [6]. The abnormal grid operation can cause considerable economic losses to the operator and end-user. Therefore, power quality problems must not be neglected [7].

Low power quality is more pronounced in distribution systems, due to the high number of variable, nonlinear and unpredictable loads connected to them. As low voltage distribution grids are supplied by distribution transformers, these can be highly affected by the low power quality presented in these systems. When operating under these conditions, the transformer suffers higher core losses, which increase its hotspot temperature. This is a critical variable, as it relates to the winding isolation degradation and, therefore, the decrease in transformer lifetime [8].

Distribution transformers can be subject to different power quality problems in distribution grids when supplying nonlinear currents and nonlinear voltages $[9,10]$. Industrial applications, such as elevators, rolling mills, arc furnaces, among others, consume high amounts of current in shorts periods, worsening distribution grid operation. Power quality issues, such as flickering, can be generated due to the operation of industrial machinery [11]. Additionally, new kinds of loads establish new operating conditions for distribution transformers and the grid, for which they were not designed. For example, high penetration of wind and photovoltaic systems connected to the grid through power converters add a degree of uncertainty to the grid operation due to the high variability of these resources and also due to the harmonics injected by the power converters [12]. The distributed characteristic of these systems makes it possible that the voltage profile on the distribution grid can increase close to the connection points under high penetration periods [13]. This scenario can represent a problem for grid control systems, such as transformer tap changers, making them switch when it is not required [14]. On the other side, the widespread of electric vehicles can overload the grid. Electric vehicles fast-charging algorithms tend to delay the vehicle charging process to the night, due to economic incentives [15]. This scenario can overload the distribution transformers, reducing their lifetimes [16]. Several methods are proposed in order to reduce the impact on distribution transformers-for example, utilizing the power converter of distributed renewable energy resources for volt-var control, smart control system, intelligent and coordinated charging algorithms and including the distribution transformer hotspot model into the power converter control systems, the use of battery energy storage systems in order to operate in periods of high energy demand, among others [16-18].

The regulating capabilities provided by a distribution transformer are limited. To deal with slow dynamic voltage variations, they are provided with on-load taps commutating systems, which allow for modifying the winding ratio without disconnecting the load. Nonetheless, these systems operate in a discrete manner and with dynamics in the order of seconds. Additionally, they are the main transformer failure cause, which jeopardizes the distribution grid and adds additional maintenance costs. The use of electronic assisted taps commutating systems is available, allowing one to decrease the commutation times and losses. Nevertheless, they suffer additional conduction losses and operate in discrete voltage steps as well [19]. Distribution transformers do not have mechanisms to mitigate load current harmonics, it being necessary to design them to operate under these conditions. These transformers are known as k-rated transformers [20].

There are several solutions based on power electronics converter to mitigate distribution grid power quality problems. Custom Power Devices (CPDs) are power electronicsbased solutions connected to the low-voltage grid that allow for mitigating for different power quality problems. Among these solutions, the Distributed Static Compensator (D-STATCOM) allows for compensating for current-based power quality problems. The Dynamic Voltage Restorer (DVR) injects a series voltage to the distribution line, which compensates for voltage disturbances. Finally, it is possible to compensate for voltage and current using a Unified Power Quality Compensator (UPQC) [6]. The effects of low power quality problems on distribution transformers, end-users, and utilities variate according to the selected solution. For example, the D-STATCOM can mitigate current harmonics 
generated by the load, reducing the distribution transformer stress. Nonetheless, the load can suffer from supply voltage disturbances, which can not be directly compensated by this unit. The latter is not the case for the DVR, which provides fast voltage correction. Nevertheless, harmonics currents generated by the load are not compensated by it, reducing the distribution transformer lifetime. As the UPQC combines both compensators in one single solution, it can provide sinusoidal voltages and currents to the load and distribution transformer, respectively. Its benefits on the distribution transformer depend on the connection points. For example, when the UPQC is connected to the primary-side, it can actively regulate the transformer voltage. Meanwhile, when it is connected to the secondary-side, the UPQC can provide harmonics mitigation and power factor correction, among others. Nonetheless, in both cases, one variable is not compensated. Additionally, unless storage systems are included, CPDs can not mitigate inrush currents generated by the transformer startup process or by sudden voltage variations. These systems are capable of mitigating a variety of power quality issues, but the distribution transformer is not fully protected from the unpredictable distribution grid operating conditions.

Solid-State Transformers (SSTs) are an attractive solution to cope with power quality problems, replacing conventional distribution transformers. They are based on power electronics, which allows for fast and precise voltage and current control [21]. Nonetheless, the efficiency of these systems is lower compared to other existing solutions, due to the necessity of operating with a high number of submodules, power conversion stages and the processing of the complete power flow. Compared to a distribution transformer, the complexity of an SST, the additional power electronics, and protection systems makes the SST a yet less reliable option [22]. In addition, SSTs are far more expensive compared to Low-Frequency Transformers (LFTs) of a similar power and voltage level. New power converter topologies, control systems, and redundancy are reducing this gap [23].

Hybrid distribution transformers (HDTs) combine a distribution transformer with one or more power electronics conversion stages, which are designed to operate at a fraction of the distribution transformer rated power. Nowadays, the available configurations, topologies, and control objectives are diverse. The HDT concept is presented in Figure 1. As can be seen, the HDT is the interface between the medium and the low-voltage grid, where the HDT is any nonspecific combination of power electronics modules with the distribution transformer [24]. Compared to other solutions, as they are based on the idea of the partial power converter, the control capabilities are limited to the power converter rating, which is typically between $10 \%$ and $20 \%$ of the transformer rated power [25]. Additionally, for the same reason, the efficiency of the power converter stages has a low impact on the overall conversion efficiency. The use of protection systems, such as bypass switches, varistors, and DC-Link clamping circuits, increases the power converter losses and volume. Nonetheless, due to the partial power converter, the protection systems have an insignificant impact on the overall efficiency and volume [26,27]. There is no restriction on the power converter location, with works reporting several different connection possibilities, i.e., medium and/or low voltage side connections, each with its own advantages and disadvantages. Therefore, the HDT configuration and power converter location will determine the power converter topology, semiconductors rating, and the provided compensation [28].

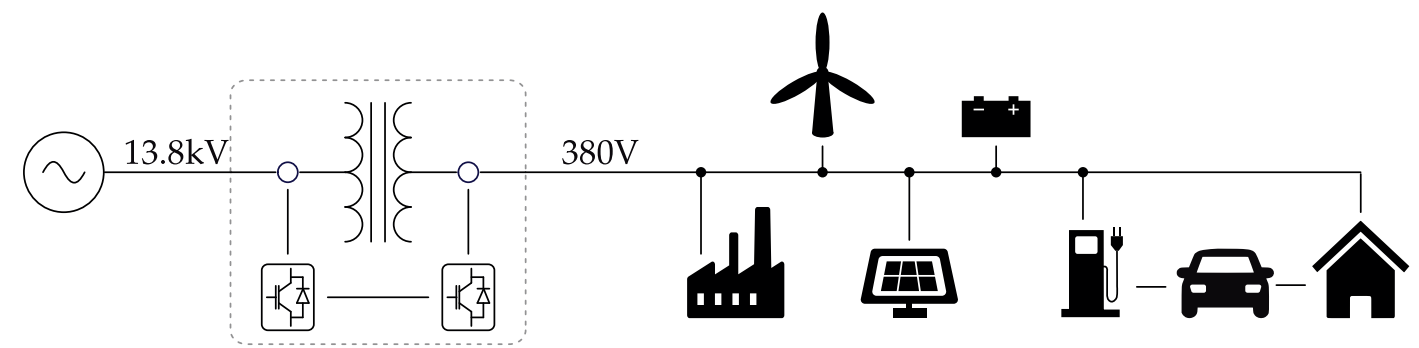

Hybrid Transformer

Figure 1. Hybrid distribution transformer (HDT) concept. 
One of the main concerns with the applicability of new technologies in the grid is power system reliability. LFT can operate for several seconds under grid failures, such as short circuits. In contrast, power converters have a very limited short circuit current supplying capacity. Therefore, solutions based completely on semiconductors, such a SSTs, are not applicable to the actual power system yet [29]. On the other hand, some HDT configurations can operate under faults, as the HDT configuration allows for the power converter to be disconnected during the fault, letting the DT supply the short circuit currents, preserving the grid reliability [26]. We must be reminded that HDTs have attracted the attention of the industry, where ABB has filed several patents relating to their control, configurations and applications [30-33].

This work is a literature review of HDTs, focusing on the different configurations and topologies available, in conjunction with the available applications or auxiliary services that it can provide.

\section{Hybrid Transformer Configurations}

HDT configurations are classified according to the energy source of the power converter unit, i.e., if the energy is obtained from a capacitor, from the LFT primary or secondary-side winding, or an auxiliary winding (AW). Simultaneously, each one can be classified according to the method utilized by the power converter to inject its energy to the grid, which can be done in series to the grid, in series with Coupling Transformers (CTs), in shunt configuration or connected to the LFT core. The HDT configurations are presented in Figure 2. The compensation provided depends on the configuration and also on the power converter topology. Nonetheless, assuming that both ends of the power converter are fully controllable, the compensation type provided by each configuration can be classified as shown in Table 1. They can be classified into shunt, series with and without $\mathrm{CT}$, and magnetic compensation. The latter can be considered as a shunt or series compensation according to the core configuration of the LFT.

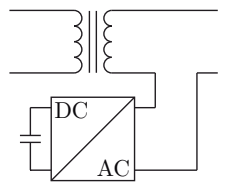

(a)

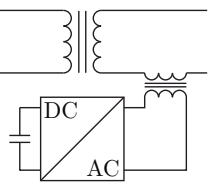

(b)

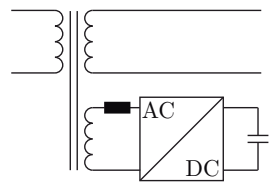

(c)

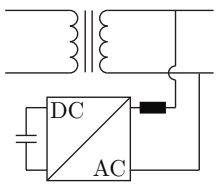

(d)

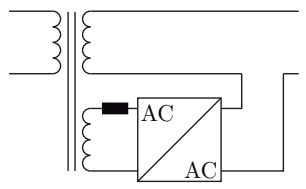

(e)

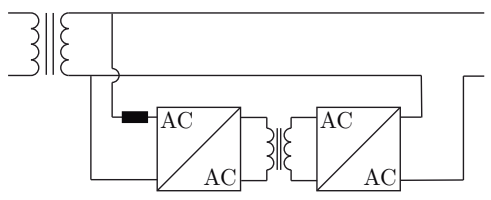

(h)

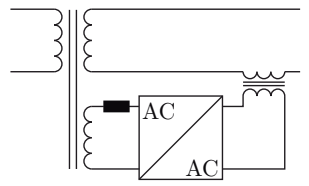

(f)

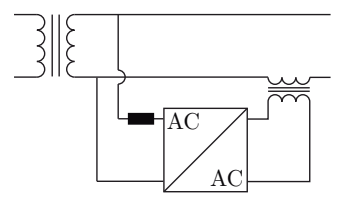

(i)

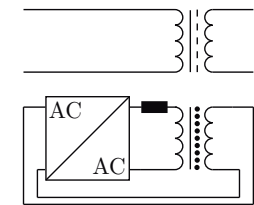

$(\mathrm{g})$

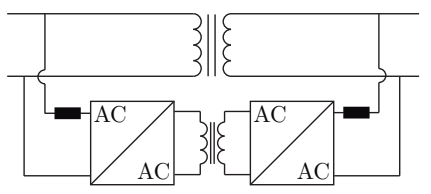

(j)

Figure 2. HDT configurations. (a) Power converter connected in series without a coupling transformer (CT). (b) Power converter connected in series with CT. (c) Power converter connected to the low-frequency transformer (LFT) core. (d) Power converter connected in shunt configuration. (e) Power converter connected to auxiliary windings (AWs) and in series without CT. (f) Power converter connected to AWs and in series with CT. (g) Power converter connected to two AWs. (h) Power converter connected to the secondary-side and in series without CT. (i) Power converter connected to the secondary-side and in series with CT. (j) Power converter connected to both sides of the LFT in shunt configuration. 
Table 1. Compensation provided by HDT configurations.

\begin{tabular}{|c|c|c|c|c|c|c|}
\hline \multirow{12}{*}{ 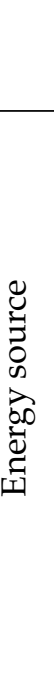 } & & & \multicolumn{4}{|c|}{ Compensation } \\
\hline & & & Series & Series $(C T)$ & Shunt & Magnetic \\
\hline & \multirow{4}{*}{ 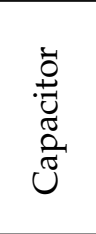 } & (a) & $x$ & & & \\
\hline & & (b) & & $x$ & & \\
\hline & & (c) & & & & $x$ \\
\hline & & (d) & & & $x$ & \\
\hline & & (e) & $x$ & & & $x$ \\
\hline & 爻. & (f) & & $x$ & & $x$ \\
\hline & 43 & (g) & & & & $\mathrm{x}$ \\
\hline & & (h) & $x$ & & $x$ & \\
\hline & $\underset{7}{7}$ & (i) & & $x$ & $x$ & \\
\hline & 5 & (j) & & & $\mathrm{x}$ & \\
\hline
\end{tabular}

In this section, HDT configurations are categorized regardless of the phase numbers of the grid. Additionally, it is only in self-supported HDTs that the conversion method is shown, whereas in the remaining configurations the power converters are generalized as AC/AC converters. In these configurations, it is assumed that the power converters operate in a decoupled manner and both terminals have a four-quadrant operating region. Later, in the analysis section, the HDT operating regions are presented in terms of the configurations and power converter topologies.

\subsection{Self-Supported Hybrid Transformers}

Self-supported HDTs, or HDTs where the energy is obtained from DC capacitors are presented in Figure $2 \mathrm{a}-\mathrm{d}$, and their compensation types and characteristics are shown in Table 1. Regardless of the power converter connection method, when utilizing DC capacitors as the energy source, the power converter is only capable of providing reactive power compensation, and an additional control method based on active power is required in order to charge and regulate the capacitor voltage to its rated value.

The configuration of Figure 2a is utilized to provide voltage regulation to the distribution grid [24]. No isolation is required when the power converter is connected between the neutral point and the LFT winding. Alternatively, if the neutral point is not accessible, it is possible to connect the power converter to the distribution line without CTs utilizing per phase power converter topologies [34]. It is possible to realize the connection to the distribution line utilizing CTs, as configuration Figure $2 \mathrm{~b}$ shows, allowing for the use of three-phase power converter topologies, instead of single-phase configurations, which reduces the converter elements. The integration of a DC source, such as a supercapacitor bank, into the DC-Link allows for voltage control utilizing active power, which extends the converter regulation capabilities [35]. Additionally, thanks to the presence of the CT, the power converter can be connected into the medium voltage grid [36]. The connection to the primary-side of the LFT allows the power converter to provide additional services to the HDT, such as voltage harmonics filtering.

It is possible to connect the power converter into an auxiliary winding of the LFT, as Figure $2 \mathrm{c}$ shows, providing magnetic compensation. In this case, the secondary-side and auxiliary winding share the same core path and, therefore, share the same magnetic flux, which is equivalent to a power converter connected in a electrical shunt configuration [24]. The same configuration has been proposed to provide current filtering in large-power industrial rectifier systems and shipboard power systems [37,38]. The auxiliary circuit can contain tuned filter branches which can be actively controlled in order to mitigate characteristics harmonics of the supplied nonlinear systems [39]. 
The configuration of Figure $2 \mathrm{~d}$ allows for providing shunt compensation by connecting the power converter to the low-voltage distribution transformer side [24]. This configuration can be integrated to the low-voltage distribution grid with or without CTs [40]. Additionally, it is possible to utilize the same configuration connected to the primary-side. Nonetheless, unless multilevel power converter topologies are utilized, CTs are required to connect the converter due to the higher voltage levels [41]. Alternatively, medium voltage semiconductors can be used for a direct connection to the medium voltage grid $[42,43]$. Yet another alternative to cope with the connection to medium voltage is to utilize the LFT taps to reduce the AC voltage connection level and the minimum required DC-Link voltage. This solution has been proposed as a Static Synchronous Compensator (STATCOM) integrated to the LFT with and without pasive filters branches [44]. Nonetheless, due to the current compensation that is made in the taps, the current distribution of the primary winding is not equal, which can cause overcurrents and damage them. Therefore, the reactive power compensation must be limited according to the load power factor in order to avoid failures [45]. The same idea has been proposed applied to the secondary-side taps, providing active filtering [46].

\subsection{Hybrid Transformers Connected to Auxiliary Windings}

Figure $2 \mathrm{e}-\mathrm{g}$ show configurations where the power converter obtains its energy from an auxiliary winding of the LFT. Their characteristics are shown in Table 1. The main characteristic of these configurations is that the presence of the auxiliary winding provides magnetic isolation, allowing one to synthesize a single-phase voltage, which can be connected in series to the distribution line. Then, depending on the system phase numbers and power converter characteristics, one of the three mentioned configurations should utilized, as explained below.

In the configuration of Figure 2e, the power converter is connected in series to the transformer secondary side when it has open windings and the neutral connection is realized on the power converter terminal. Most of research studies have been focused on this configuration and different power converters have been proposed for it. Additionally, several patents that utilize this configuration have been filed by ABB [30-33]. HDTs based on unidirectional power converters have been proposed. Nonetheless, their operation region is highly dependent of the grid and load conditions [47]. On the other hand, solutions based on Back-to-Back (B2B) AC/DC power converters extend the regulation capabilities, allowing one to provide voltage and current control in a broader range [48]. Additionally, it is possible to utilize AC/AC choppers converters. Nonetheless, the regulation capabilities depend on the utilized power converter topology and on the grid impedance matching. Additionally, depending on the converter topology, under grid faults and after bypassing the power converter, the LFT is unable to provide rated voltage due to unconventional ratios of the secondary and auxiliary winding. These issues can be solved utilizing bipolar AC/AC power converters [49]. Another alternative is to utilize three-phase auxiliary winding for each output phase in conjunction with DC-Link based power converters. In this case, independent DC-Links per phase are required, which at the same time allows for connecting the power converter in series to the distribution line without CTs after the LFT output terminals, since the three-phase output voltages are magnetically isolated between them. Moreover, different winding configurations can be utilized in order to mitigate harmonics currents generated by the power converter switching process [50].

In the configuration of Figure $2 \mathrm{f}$, the series converter is integrated into the distribution grid by means of a CT. The auxiliary winding in conjunction with the CT isolates the power converter from the grid, which allows us to optimize and reduce the DC-Link voltage independently of the series converter location. There are solutions based on B2B AC/DC converters [28], as well as based on AC chopper converters [51,52]. Alternatively, to protect the LFT from grid voltage disturbances, it is possible to connect the series converter to the primary-side, which allows the power converter to adapt to the rated voltage level, thanks to a CT, and provide the required mitigation on the medium voltage grid [53]. 
A detailed model and compound control scheme has been presented for this configuration. The model accounts for the voltage and current disturbances, aiming to improve the system's robustness [54]. Additionally, the use of controllable tuned filter branch has been proposed for the shunt converter in order to mitigate characteristics harmonics the power converter can actively regulate the filter impedance for harmonics mitigation purposes [55].

An alternative to previous configurations, known as Power Electronics Integrated Transformer (PEIT) and Custom Power Active Transformer (CPAT, in which the power converter is completely integrated to the LFT), is shown in Figure 2g. In this case, the power converter takes its energy from an auxiliary winding, which, after being processed, is injected back to the LFT through an additional auxiliary winding. The core and winding configuration determine the purpose of the power converter. Two equivalent parallel electrical circuits can be realized by means of two windings sharing the same magnetic core path, whereas a series electrical circuit is made by a winding attached to a third core leg or a shunt magnetic core path [56]. A single-phase PEIT has been proposed, whereas the three-phase alternative has been proposed by connecting three single-phase PEITs [57]. In order to increase the level of integration, a shell type structure has been proposed, sharing the transformer yokes, which finally reduces the transformer footprint [58]. This configuration can be utilized as a power flow controller while, at the same time, can be utilized to provide current and voltage harmonics mitigation [59].

\subsection{Hybrid Transformers Connected to Transformer Windings}

Configurations of Figure 2h-i show HDTs where the power converters obtain their energy from the secondary or primary-side windings of the LFT. Their characteristics are shown in Table 1. Since the power converter is directly connected to the transformer winding, an isolation stage is required to synthesize an isolated series voltage. As shown in the presented configurations, this task can be done by means of CTs or by internal-isolated power converters.

In the configuration of Figure $2 h$, the power converter takes its energy from the secondary-side winding and after processing it, it is injected in series to the distribution line. The transformerless series connection is possible due to an internal isolation stage based on High-Frequency Transformers (HFTs). This configuration has been proposed for a single-phase HDT, which, compared to solutions based on CT and connected to auxiliary windings, allows for a reduction in weight and volume, while being a retrofit and scalable solution [60-63].

The configuration of Figure 2i provides current and voltage compensation on the low-voltage side. This configuration requires the use of CTs to allow the injection of a series voltage to the distribution line. This configuration is proposed based on Back-to-Back (B2B) DC-Link based power converters [48], and with three-phase direct AC/AC converters as well [64]. Alternatively, the series converter can be placed on the primary side of the LFT utilizing CTs. Besides the isolation purpose of the CTs, in this scenario they are required in order to allow for the connection to medium voltage and operate with low voltage DC-Links [65]. Alternatively, when the power converter is supplied by a DC system, such as a microgrid, the series and shunt converter can extract active power from it and utilize it for grid voltage and current regulation. This configuration can be realized by employing a middle isolated DC/DC stage. In this scenario, the CTs are not strictly necessary, due to the isolation provided by the HFT. Nevertheless, CTs allow for reducing the required number of DC/DC converter submodules or for the converter design to employ low voltage rated semiconductors [66].

In Figure $2 \mathrm{j}$ the power converter is connected to the primary and secondary-side in shunt configuration utilizing power converters isolated by HFTs. For example, this configuration is utilized for the integration of renewable energy systems or when it is desired to increase the distribution transformer capacity without replacing it. In principle, this configuration does not allow us to provide series voltage regulation directly; nonetheless, it can be controlled indirectly through reactive power injection. A three-phase converter 
utilizing isolated DC/DC converter is proposed, as well as a single-phase isolated AC/AC converter $[67,68]$.

\subsection{Three-Stage Configuration}

The configuration of Figure 3 is formed by a combination of two configurations presented previously: (1) It connects the primary and secondary-side by means of shunt converters utilizing HFTs, similar to the configuration of Figure 2j. (2) A series converter is connected to the distribution line, as shown in Figure 2i. In this case, and in order to provide voltage regulation to the LFT, the CT is located on the primary-side [69].

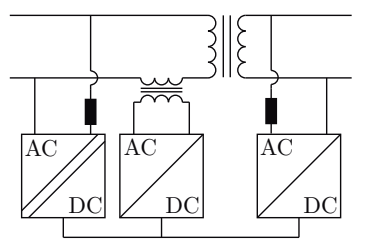

Figure 3. Combined HDT configuration.

\subsection{Additional HDT Configurations}

In this section, HDT configurations that cannot be classified easily with the previous classification are presented in Figure $4 \mathrm{a}-\mathrm{c}$.

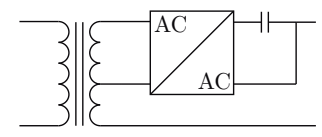

(a)

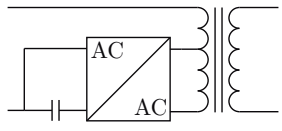

(b)

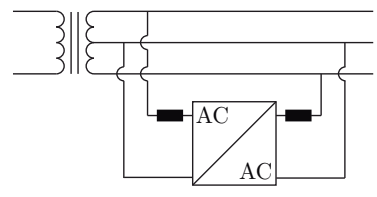

(c)

Figure 4. Additional HDT configurations. (a) Series connection to the high voltage taps. (b) Series connection to the low voltage taps. (c) Shunt connection to both phases of a single-phase with central tap grid.

In the configuration of Figure 4a, the power converter is connected to the secondaryside high voltage-side taps of the LFT. This configuration is proposed utilizing single-phase $\mathrm{AC} / \mathrm{AC}$ converters per phase and it was first presented as a controllable network transformer, which can provide power flow control between transmission lines. The nature of the $\mathrm{AC}$ chopper converters preserves the phase of the input voltage, without allowing us to modify the output voltage phase. Nonetheless, utilizing techniques based on harmonics injections, it is possible to shift the fundamental voltage to provide power flow control and act as a active power filter as well $[70,71]$. The same idea can be applied in Figure $4 b$, where the power converter is connected to the primary-side and neutral-side taps. Compared to previous configurations, this solution presents lower conduction losses since the converter is located on the primary side. When utilizing AC chopper converters, it is necessary to utilize switches to commutate between boost and buck mode for sag and swell mitigation, respectively [72]. Alternatively, DC-Link-based converters can be utilized. In this case, the taps-side converter rectifies the tap voltage, then the end-side converter inverts it in order to generate the required $\mathrm{AC}$ voltage and injects it in series to the grid. No switches are required due to the fact that the capabilities of DC-AC converters to shift its output voltage waveform [73]. In previous configurations, load harmonics mitigation is not provided, which can affect and diminish the LFT lifetime.

The configuration of Figure $4 \mathrm{c}$ is proposed exclusively for low-voltage distribution systems with central-tap. The power converter is connected between both phases to provide shunt compensation. Additionally, it allows for active and reactive power sharing between both phases for balancing purposes, reducing the neutral currents [72]. The shunt units are capable of providing power factor correction and protecting the LFT from load harmonics. 


\section{Power Converter Topologies Employed for Hybrid Transformers}

The classification of the power converter topologies utilized in HDTs proposed in the literature are summarized in Figure 5. The topologies can be classified into DC/AC and $\mathrm{AC} / \mathrm{AC}$ converters. The former are power converter utilized in self-supported HDT configurations, exclusively. The latter are composed of power converters with or without energy storage systems, which include the AC choppers and matrix converters, DC-Linkbased power converters and power converter topologies with galvanic isolation-based Dual-Active Bridges (DABs) and Solid-State Transformers (SSTs). With the advent of improved medium-voltage wide band gap devices [42,74-76], direct medium-voltage conversion is now a feasible reality. Although in nascent stages, several works have introduced direct medium voltage $\mathrm{AC} / \mathrm{AC}$ and $\mathrm{AC} / \mathrm{DC}$ converters $[43,62,77,78]$.

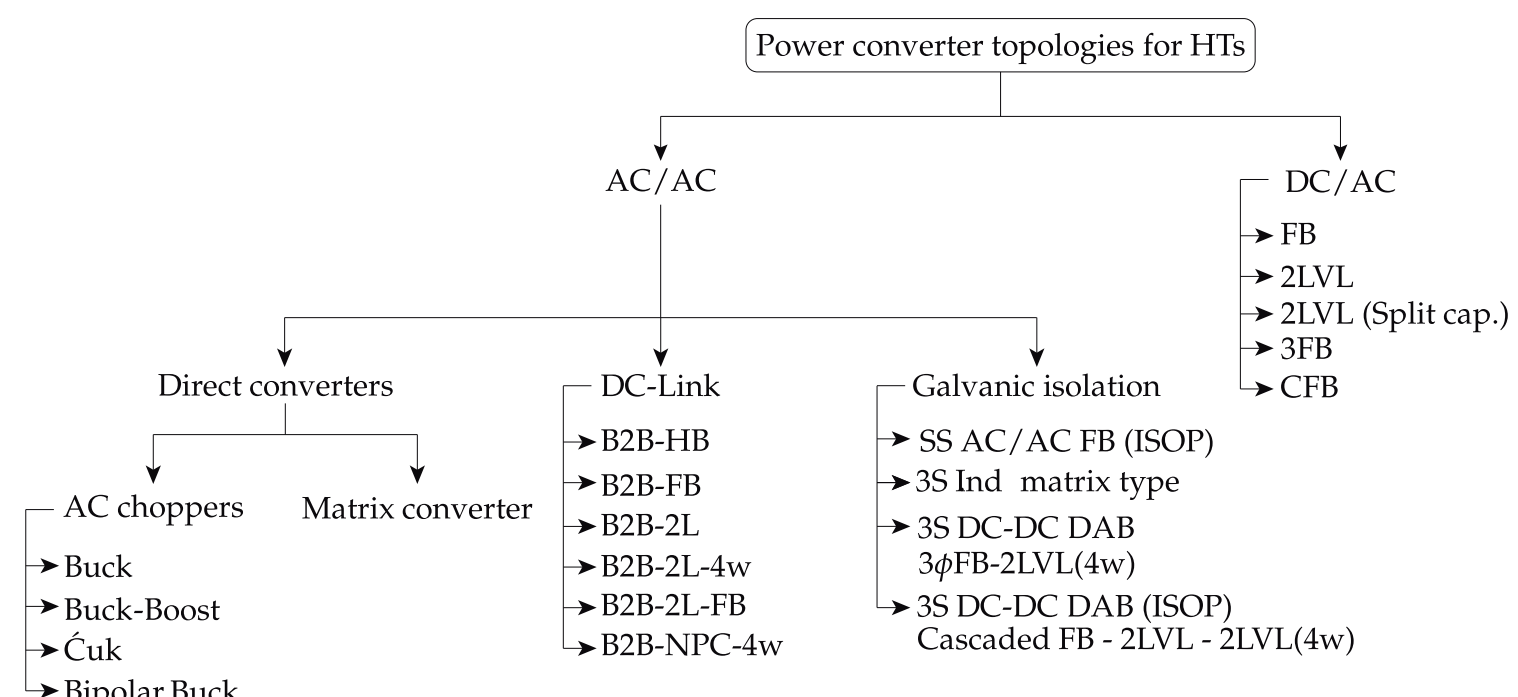

Figure 5. Power converter topologies.

\subsection{DC/AC Power Converter Topologies}

The power converter topologies presented in Figure 6 are based on DC/AC converters, and they are utilized in self-supported configurations, exclusively. For the configuration of Figure $6 \mathrm{~d}$, the three-phase two-level converter (3P2L) of Figure 6a is utilized to provide current-based mitigation. The connection to the low-voltage grid allows for the use of low-voltage semiconductors [6]. Alternatively, this topology can be connected to the medium-voltage grid by means of the LFT ground-side taps in order to reduce the voltage connection level [46]. If the LFT taps are not available, it is possible to utilize the same topology, but connected to the medium-voltage grid by means of CTs [6,41], or by using medium voltage switches. Similarly, the same power converter can be utilized to provide voltage-based compensation, considering a capacitive output filter, as shown in Figure 6b. CTs are also required in order to magnetically isolate the output phases and connect them in series to the distribution line. Slightly modifications of the previous topology are available, such as splitting the DC-Link capacitor to provide a two level bipolar voltage at the converter output terminals, as shown in Figure 6c. This topology is utilized in Dynamic Voltage Restorers (DVRs) coupled to the distribution grid by means of $\mathrm{CTs}$, as configuration of Figure 6b [35]. Alternatively, three FB (3FB) converters connected to a common DC-Link capacitor can be utilized to provide a three-level output voltage, as shown in Figure 6d. Similar to previous topology, in order to connect it in series to the distribution line, it is required to utilize CTs, due to the fact that the output phases of the power converter are not magnetically isolated [36]. The power converter topology of Figure 6e corresponds to three FB converter with independent DC-Links. In terms of power 


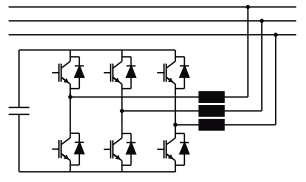

(a)

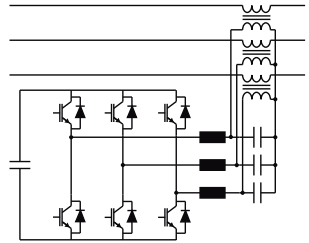

(b)

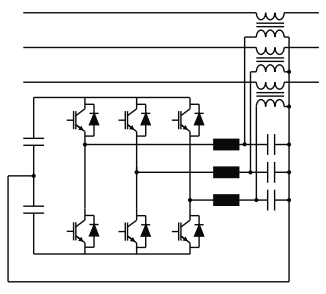

(c)

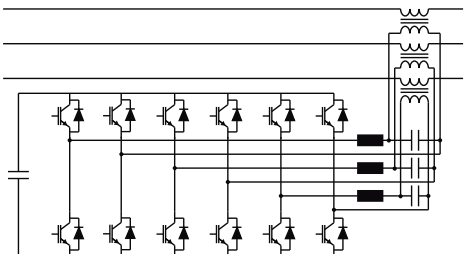

(d) quality, FB converters allow one to generate a three-level output voltage. This topology is utilized in the configuration of Figure 2a to provide series voltage compensation [79].

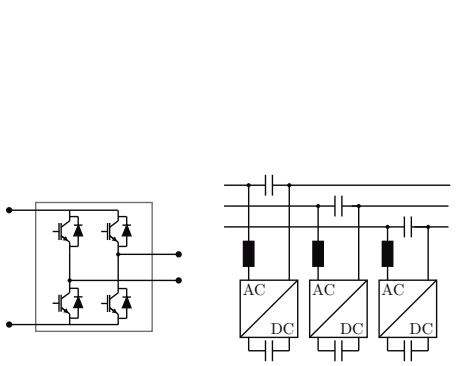

(e)

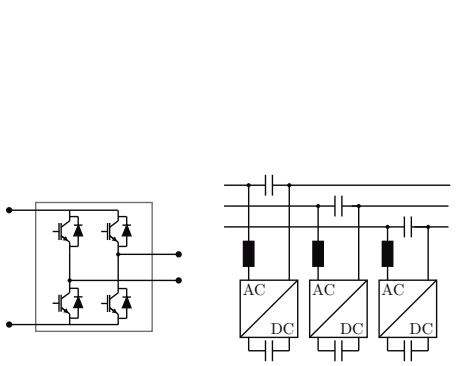

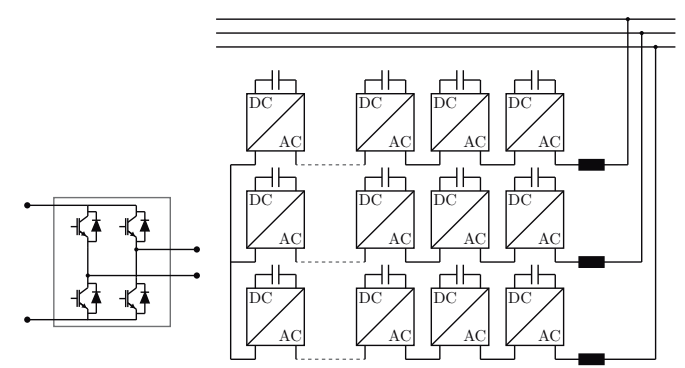

(f)

Figure 6. DC/AC power converter topologies. (a) Three-phase two-level converter. (b) Three-phase two-level converter with CTs. (c) Three-phase two-level converter with split capacitor and CTs. (d) H-bridge converter per phase with common DC-Link. (e) H-bridge converter per phase with independent DC-Link. (f) Cascaded H-bridge converter.

The topology of Figure $6 \mathrm{f}$ corresponds to a cascaded FB (CFB) multilevel converter. The series connection of HB modules allows for the utilization of low-voltage semiconductors in order to increase the converter voltage. Among other modulation techniques, when utilizing Phase-Shifted PWM (PS-PWM) and $n$ number of submodules per phase, the converter is able to generate an output voltage with $2 n+1$ levels, improving the power quality and diminishing filtering requirements [80]. This topology has been proposed for converters connected in shunt configuration to the medium-voltage grid, such as in Figure $2 \mathrm{~d}$. In this case, the converter is connected to the ground-side taps of the LFT [81]. Compared to other alternatives, this modular solution is easily expansible in terms of power requirements, requiring one to connect the power converter to a higher voltage tap and increase submodules number [45].

\subsection{AC/AC Power Converter Topologies}

In this section, HDT topologies based on AC/AC power converters are presented. Four categories of power converters are introduced, which are AC choppers, shown in Figure 7, direct power converters, shown in Figure 8, power converters based on DC-Link stages, shown in Figure 9, and power converters based on SSTs, shown in Figure 10.

\subsubsection{AC/AC Converters Based on Direct Converters}

The converter topologies of Figure 7 are AC chopper converters [82]. In this category, the AC buck, Buck-Boost, Cuk and bipolar Buck converter have been proposed in HDT applications. These topologies are employed in configurations of Figure $2 \mathrm{e}$, and they are not capable to provide current regulation, as the power converter topology is incapable of controlling the phase of its output voltage, which depends on the grid voltage phase and power converter filter characteristics. On the other hand, the delivered active and reactive power mainly depends on the load power factor. Considering an ideal lossless power converter, the output voltage for the topology presented in Figure 7a has a linear relation with the duty cycle $[49,83]$, and the output voltage for topologies presented in Figure $7 \mathrm{~b}, \mathrm{c}$ have a non linear relation. The output voltage gains are summarized in Table 2 . Due to the 
interaction with the filters and load impedances, it is required to limit the power converter duty cycle. Furthermore, the converter voltage gain of the three mentioned converters are highly dependent on the filter and load impedance matching, which is difficult to guarantee in distribution systems. Nonetheless, the effective operating region of the AC buck-boost chopper is larger than the other two alternatives [84].

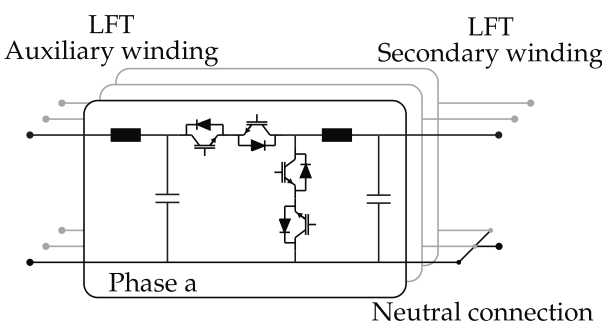

(a)

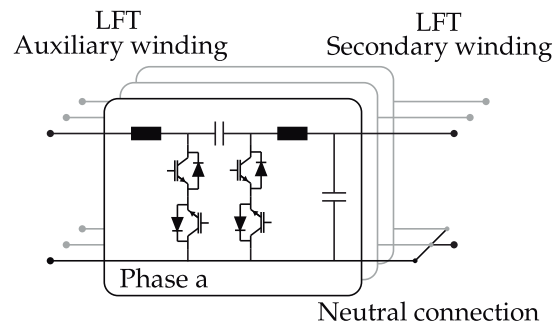

(c)

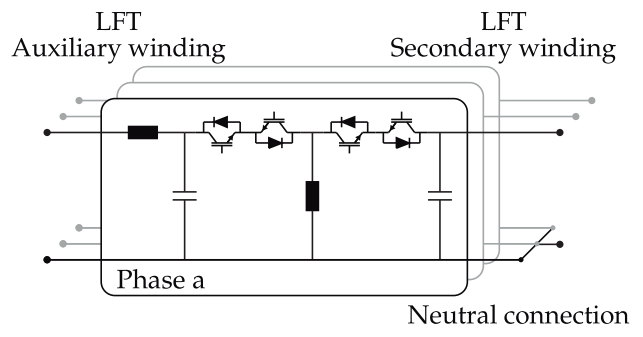

(b)

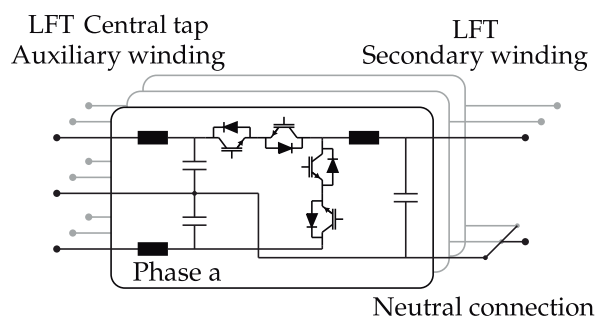

(d)

Figure 7. Single-phase representation AC choppers. (a) AC buck converter. (b) AC buck-boost converter. (c) AC Ćuk converter. (d) AC bipolar buck converter.

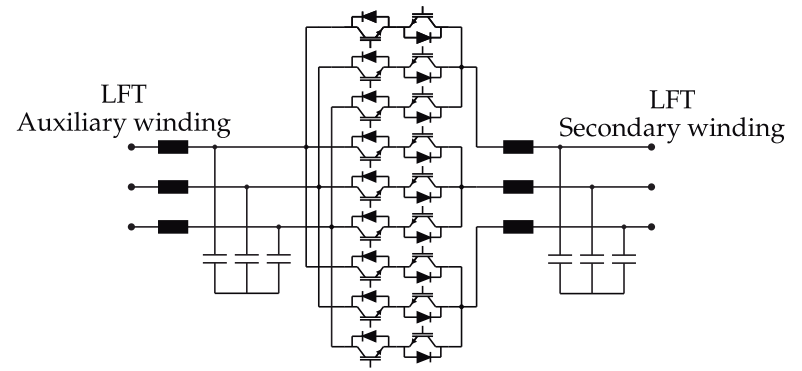

Figure 8. Direct matrix converter.

Table 2. AC choppers ideal voltage gain.

\begin{tabular}{cc}
\hline Power Converter & Ideal Voltage Gain \\
\hline AC Buck & $D$ \\
\hline AC Buck-Boost & $\frac{1}{1-D}$ \\
\hline AC Ćuk & $\frac{1}{1-D}$ \\
\hline AC bipolar Buck & $2 D-1$ \\
\hline
\end{tabular}

In the previous three configurations, the LFT secondary and auxiliary winding must be designed with unconventional ratios in order to provide sag and swell mitigation. Therefore, the HDT is unable to operate successfully when the power converter is bypassed, for example in the case of faults, as the LFT is not capable of providing the rated voltage [85-87]. An alternative to previous topologies is the bipolar buck converter of Figure $7 \mathrm{~d}$. This solution requires the use of a center-tap auxiliary winding, allowing one 
to generate an output voltage which is formed by the commutation of the input voltage and its counter-phased waveform. With a proper commutation, it is possible to generate in-phase and counter-phase output voltages, allowing one to compensate for sags and swells without requiring unconventional winding ratios or mode-selecting switches. Therefore, this topology is compatible with fault-operation modes. Nonetheless, these features come at the expense of a LFT with center-tap auxiliary winding, increasing the volume and weight of the LFT [88].

In the presented solutions, the required switch type depends on the modulation method utilized for the power converter [89]. Unidirectional switches can be utilized for zero-sequence modulation schemes. In these cases, the output voltage is proportional to the input voltage, according to its voltage gain characteristic. Since the switches are commutated simultaneously, reduced switch configurations can be utilized [90,91]. On the other hand, a certain degree of phase control can be provided if harmonic injection methods are utilized, such as Dual Virtual Quadrature Sources (DVQS), which increases the power converter regulation capabilities [92]. In these cases, bidirectional switches are required [89].

In Figure 8, a matrix converter is presented. In this topology, each input phase can be connected to an output phase through bidirectional switches. Typically, inductive loads are also supplied; therefore, it is mandatory that no open circuits are generated on the output circuit. Additionally, in order to provide a current path during commutations, capacitive filters are required on the input-side. Input and output LC circuits can act as a current and voltage harmonics filtering as well. Additionally, it is forbidden to connect two or more input phases to the same output in order to avoid short circuits [93]. This topology is employed in the configuration of Figure 2e, allowing one to utilize the conventional secondary-side winding ratio, as the output voltage phase can be controlled independently of the grid voltage phase. The auxiliary winding ratio depends on the required compensation level, considering that the maximum matrix converter voltage gain is reduced by a factor of $\sqrt{3} / 2$ [94]. This topology can be also applied on the configuration of Figure 2i [64]. In this case, the converter input is connected in shunt configuration to the secondary winding and in series to the low-voltage distribution line by means of CTs. Compared to previous power converter topologies, three-phase direct matrix converters can control both the output voltage phase and frequency, allowing for a broader regulation capability, while providing input power factor factor correction [95].

\subsubsection{AC/AC Converters with a DC-Link Stage}

Topologies presented in Figure 9 consist of an AC/AC power converter with intermediate DC/AC converters connected by a common DC-Link. Compared to previous $\mathrm{AC} / \mathrm{AC}$ converter solutions, the presence of the DC-Link allows one to decouple the rectifier and inverter stage from the control system point of view [93]. Different power converter topologies can be utilized in each stage, but the following has been proposed for HDT applications up to date. The topology of Figure 9a is a back-to-back half-bridge (B2B-HB) converter with split capacitor, generating a two-level bipolar output voltage, and it is utilized in configurations of Figure $4 \mathrm{~b}, \mathrm{c}$. This topology allows us to control the output voltage magnitude and phase, realizing reactive power control and providing power factor correction. When utilized in central tap low voltage grids, the DC-Link presence allows us to exchange active power between phases for balancing purposes [72].

The topology of the three single-phase B2B FB (3-B2B-FB) converter of Figure 9b is employed in configurations where the power converter is integrated through the auxiliary winding to the distribution transformer and connected in series to the grid without CTs, as in Figure 2f. Regarding other per-phase topologies, the H-bridge converter is simple, reliable and has low losses. This solution has been proposed when connected to the secondary and primary-side, though the efficiency when it is connected to the low-voltage grid is slightly higher [28]. 


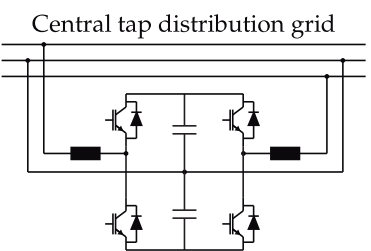

(a)

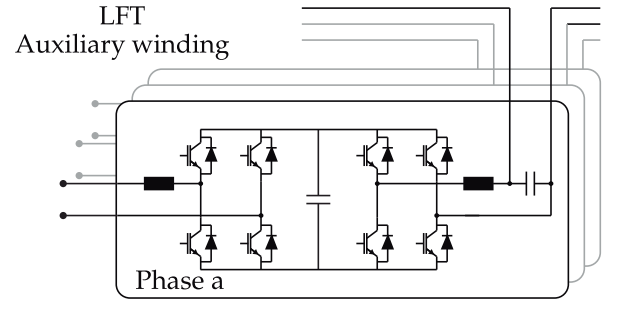

(b)

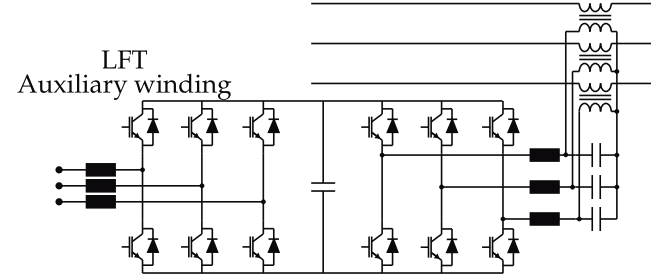

(c)

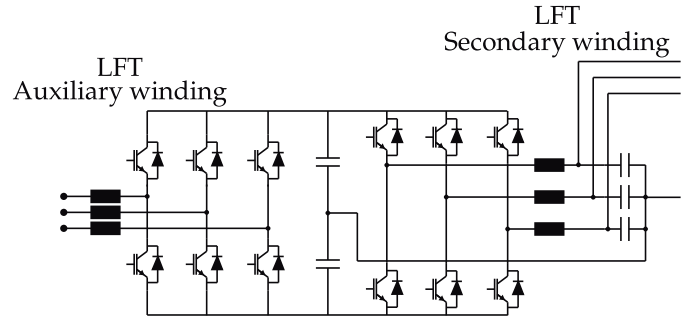

(d)

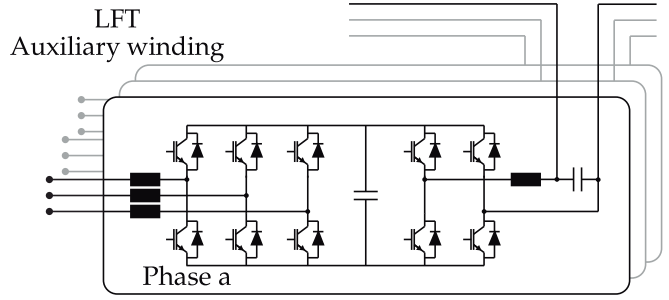

(e)

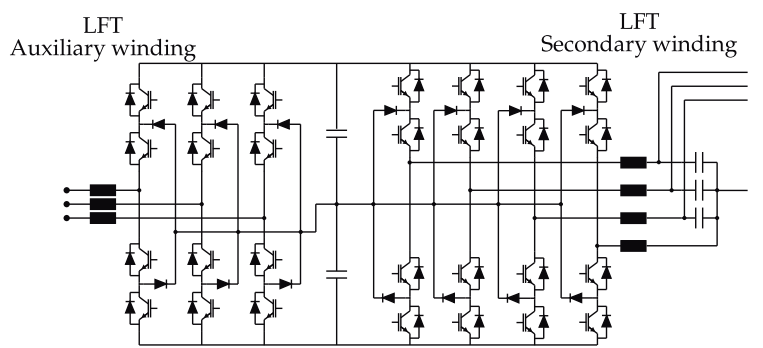

$(\mathbf{f})$

Figure 9. AC/AC converter with intermediate DC-Link stage. (a) Back-to-back half-bridge converter. (b) Back-to-back H bridge converter. (c) Back-to-back three-phase two-level converter. (d) Back-to-back three-phase two-level converter for four-wire grids. (e) Single-phase representation of a three-phase two-level converter and H-bridge converter in back-to-back configuration. (f) Back-to-back neutral pointed clamped (NPC) converter for four-wire grids.

The back-to-back three-phase two-level converter (B2B-2L) of Figure 9c is the most recurrent topology. In terms of losses and semiconductor costs, this topology integrated to the grid with a CT has the best performance when it is compared to other three-phase converters without $\mathrm{CT}$, such as the three single-phase B2B-FB, the three-level converter with and without intermediate DC/DC converters and matrix converters [28]. This converter is employed in configuration of Figure 2f,i, where the use of CTs also allows for integration to the medium-voltage side without recurring to multilevel alternatives or the use of medium voltage switches [65]. In a similar manner, it is possible to utilize it in configuration of Figure $2 \mathrm{~g}$ since both ends of the power converter are connected to auxiliary windings, which are designed to comply with the converter rating requirements [59]. In cases when the shunt converter is connected to four-wire grids, the neutral wire can be obtained from the middle point of the split DC-Link capacitor, as Figure 9d shows $[48,96]$. In this case the rectifier-end is connected to an auxiliary winding, while the inverter-side is connected in series to the secondary-side of the LFT, and the neutral connection is provided by the split capacitor. An alternative topology utilized in the configuration of Figure 2e is presented in Figure 9e. A three-phase two-level converter shares a common DC-Link with an FB converter (B2B-2L-FB). The FB is connected in series to the distribution line and therefore three independent converters are required in order to be utilized in three-phase grids without requiring CTs. The rectifier ends, given by the three-phase two-level converters are connected to independent auxiliary windings [50]. 
The B2B Neutral Pointed Clamped (NPC) converter is shown in Figure 9f. Each leg of this converter is made by four switches and two diodes, which are connected to central point of a split capacitor. Compared to two-level converter, the NPC allows to generate one additional voltage level, aiming to improve the voltage power quality [80]. This converter is employed in the configuration of Figure 2e, designed to be applied in transmission level applications, but has also been tested at distribution levels. The fourth leg of the inverter stage is utilized in order to operate under unbalanced conditions [97].

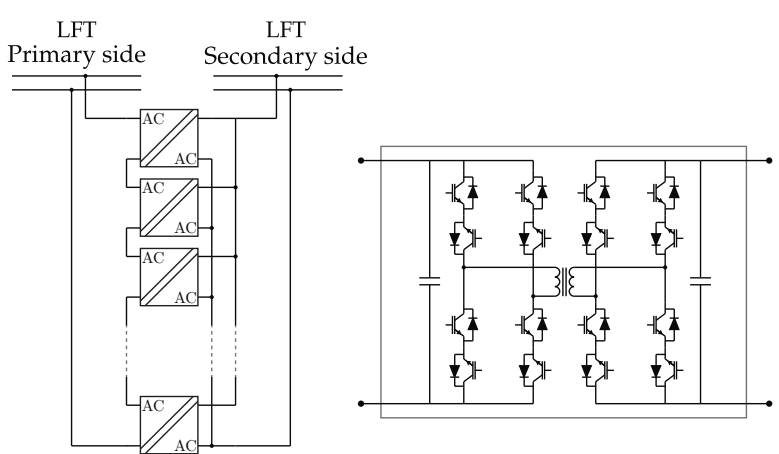

(a)

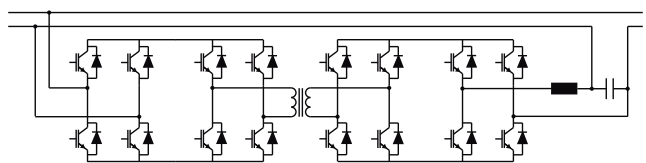

(b)

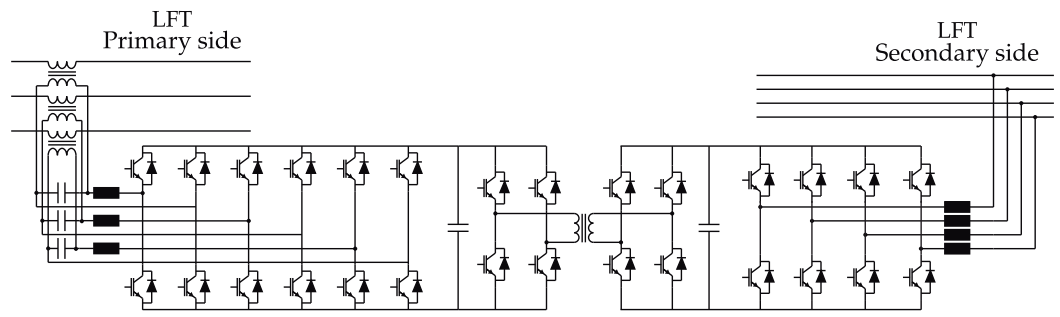

(c)
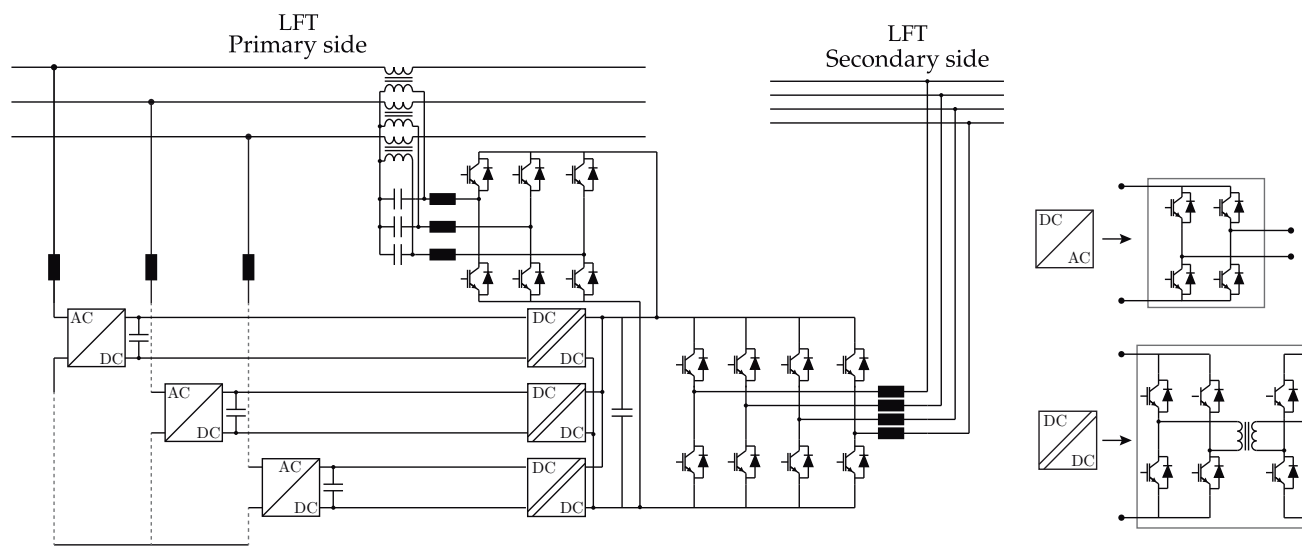

(d)

Figure 10. Power converter topologies with galvanic isolation. (a) Three-stage power converter with DAB DC/DC converter. (b) Three-stage power converter based on a indirect matrix converter. (c) Single-stage cascaded AC/AC full-bridges in input series and output parallel (ISOP) configuration. (d) Three-stage power converter with dual-active bridge (DAB) in ISOP configuration for series and shunt (four-wire) compensation.

\subsubsection{AC/AC Converters with Galvanic Isolation}

The galvanic isolated power converters are presented in Figure 10. These converters can be classified according to the number of conversion stages between the input and output AC voltages. Additionally, when multiple submodules are utilized, they can be classified according to their connection methods. In Figure 10a a single-stage Input Series and Output Parallel (ISOP) converter is presented. This topology is employed in the configuration 
of Figure 2j. In order to allow its connection to medium-voltage, a series connection of submodules is required. Then, to cope with the high currents of the low-voltage side, the submodules are connected in parallel configurations, sharing the current demand [68]. Each submodule is made of an AC-AC FB converter, and the power transfer between the primary and secondary-side of the HFTs is controlled by means of the phase-shift between both HB $[98,99]$.

The power converter topology of Figure 10b has been applied in the configuration of Figure $2 \mathrm{~h}$. This converter has been applied in single-phase grids and the presence the HFT allows one to connect the SST in series to the distribution line without requiring CTs, which reduces the weight and volume of the converter. Since the converter is placed on the secondary-side, a single-module solution is enough to cope with the connection voltages $[62,63,100]$. It is based on an indirect matrix converter and is composed of three conversion stages: (1) A folding stage, that rectifies the input AC voltage. (2) A high-frequency chopping stage. (3) An unfolding stage, that reshapes the output AC voltage [22,62]. In addition the work presented in [62], this convertor utilizes $7.2 \mathrm{kV} \mathrm{SiC}$ switches, enabling a direct a medium voltage conversion, suitable for distribution side application. In Figure 10c, an alternative three-stage power converter is presented, which is based on a single Dual-Active Bridge (DAB) converter. The DAB is utilized between the input and output stages. This topology is utilized in the configuration of Figure 2i [66]. The CTs allow for the reduction in the voltage requirement of the FB to avoid the use of multilevel topologies. Additionally, the CTs enable the use of just a single DAB converter.

In Figure 10d an alternative power converter is presented, which is based on DAB converters. This topology is utilized in configuration of Figure 3. In order to enable a medium voltage connection, a CFB multilevel converter is utilized, where the submodules are connected in an ISOP configuration to the main DC-Link capacitor. The multilevel converter supports the operation of the primary-side series converter and the secondaryside shunt converter. The series converter is based on a three-phase two-level converter, which is connected to CTs to provide magnetically isolated series voltages. On the other hand, the secondary-side shunt converter is based on a four-leg two-level converter in order to support unbalanced conditions. No CTs are required in this stage, as the voltage stress is low enough for the converter to support [69].

\section{Analysis of Hybrid Transformers}

\subsection{Operating Region}

The power converters of HDTs can operate in three different operating regions, which depends on the HDT configurations and the utilized power converter topology. The operating of each proposed topology up to date is summarized in Table 3 . The operating regions can be classified into: (1) Reactive power injection. (2) Restricted active and reactive power injection. (3) Unrestricted active and active power injection. For exemplification purposes, it is assumed that the converters are connected to the secondary-side, where the secondary winding voltage is $V_{s}$, the load voltage is $V_{L}$, the load current is $i_{L}$, the secondary-winding current is $i_{s}$, the injected shunt converter current is $i_{s h}$, and the injected series voltage is $V_{s e}$. Nonetheless, the analysis can be carried out for power converters connected to the primary side as well. 
Table 3. Power converters operating regions.

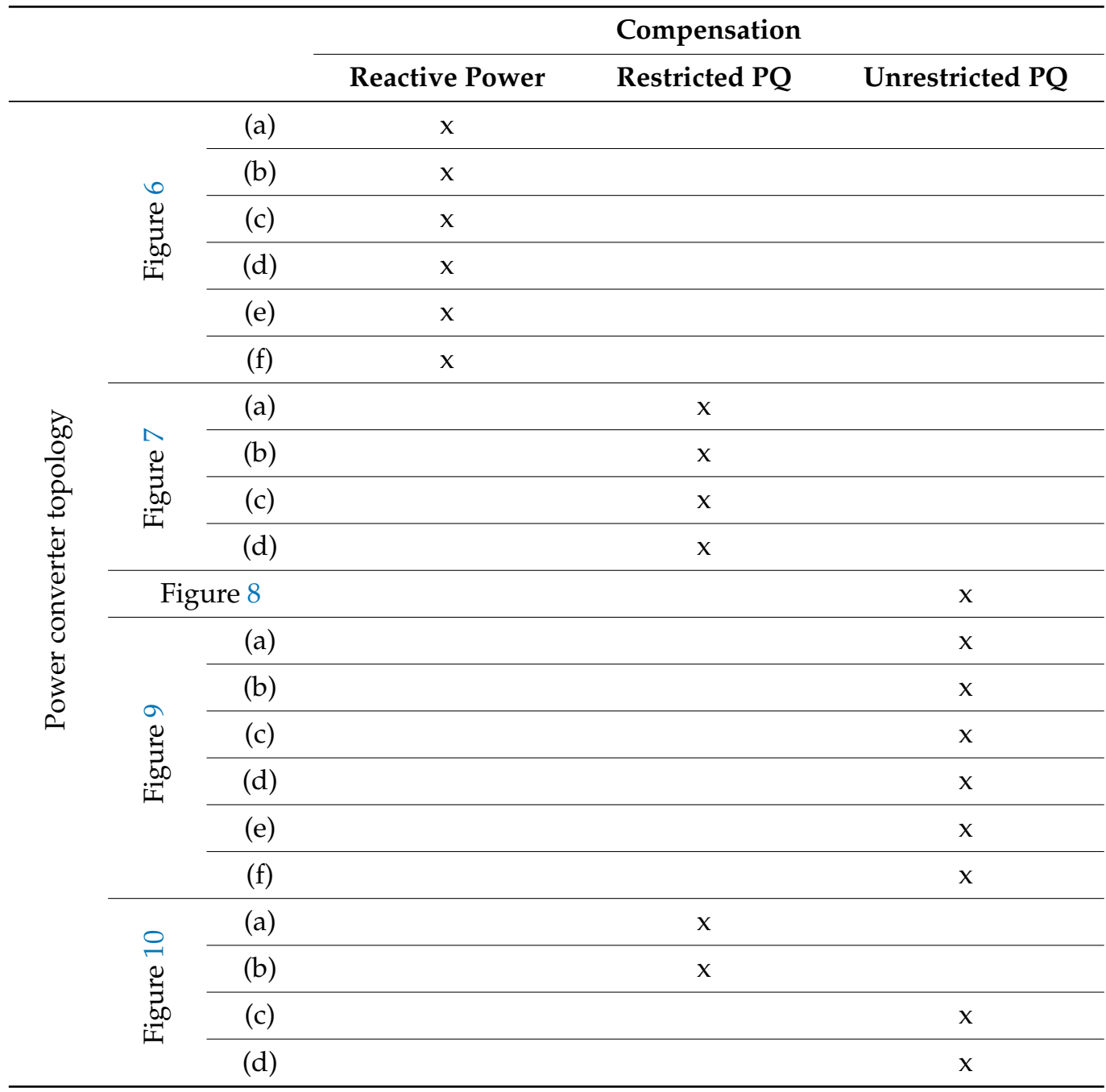

\subsubsection{Reactive Power Injection}

In the first operating region, shown in Figure 11, the power converter is only capable of injecting reactive power, which is represented by a vertical line in the PQ plane of Figure 11a. Subsequently, the total HDT output power is given by the vector sum of the delivered power of the LFT and the power converter. This operating region corresponds to self-supported configurations, utilizing topologies of Figure 6 and the energy injection method depends on the HDT configuration. For series-connected power converters, the energy is injected by means of a series voltage to the distribution line. This scenario is represented in Figure 11b. Assuming a lossless power converter, such that there is no active power consumption, the power converter must synthesize an output voltage in quadrature to the load current in order to provide reactive power. Whether the use of the series converter is utilized to regulate the primary-side or load voltage to their rated values, the injection of a series voltage modifies the angle of the grid voltage, modifying its power factor. This is represented in Figure 11b, where two different compensating voltages are applied, generating two different grid voltage phase angles.

On the other hand, for shunt-connected power converters, depending on the point of connection, a current in quadrature to the connection point voltage must be supplied. This is represented in Figure 11c. The injection of a current that controls the reactive component of the source current modifies the equivalent power factor in a similar manner to a STATCOM. 


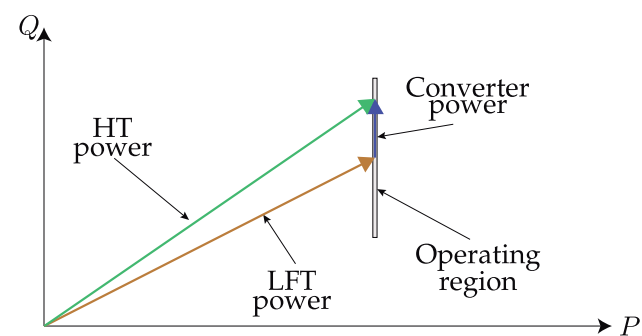

(a)

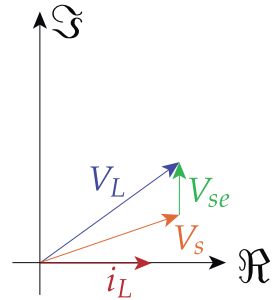

(b)

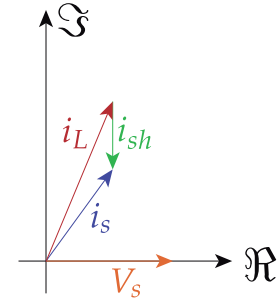

(c)

Figure 11. Operating region of HDTs whose power converters inject reactive power, exclusively. (a) Active and reactive power (PQ) operating region. (b) Series voltage injection. (c) Shunt current injection.

\subsubsection{Restricted Active and Reactive Power Injection}

In the operating region of Figure 12, the power converter is able to inject active and reactive power. Nonetheless, the apparent power angle mainly depends on the load power factor and also on the passive components utilized in the converter. This is represented in Figure $12 \mathrm{a}$, where the converter operating sector ideally corresponds to a varying angle line given by the diameter of the overall operating region. Figure $12 b$ shows the operating region in the complex plane when the power converter is utilized to provide voltage regulation. If the filter effects of the power converter are not neglected, the equivalent voltage phase of the load is modified, affecting the grid power factor.

Depending on the converter topology, the HDT will be able to add or subtract a voltage vector. This operating region occurs for the AC choppers' power converters of Figure 7, which are utilized in HDTs that obtain their energy from an auxiliary winding and inject it back to the grid in series without CTs, as shown in Figure 2e. This mode of operation is possible in two SST topologies. In the topology of Figure 10a, which is connected in parallel to the LFT as a configuration of Figure 2j, and the topology of Figure 10b connected in series to the distribution line, as a configuration of Figure $2 \mathrm{~h}$. The system is modulated to ensure that the voltage at the power converter terminals preserves the phase and angle of the supply voltage $[60,68]$.

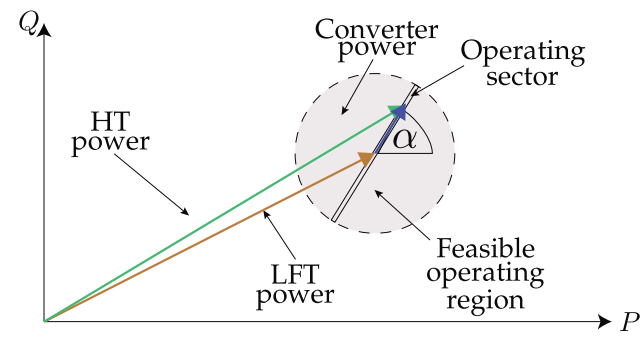

(a)

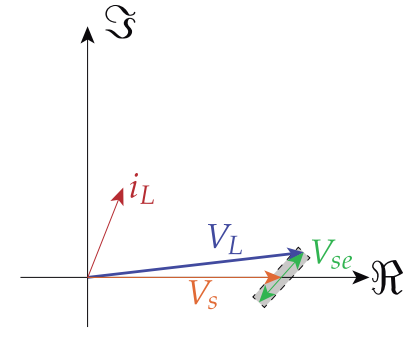

(b)

Figure 12. Operating region of HDTs whose power converters are capable to inject restricted active and reactive power. (a) PQ operating region. (b) Complex plane operating region for voltage compensation.

\subsubsection{Unrestricted Active and Reactive Power Injection}

In Figure 13, the operating region where the power converter is able to inject apparent power with an unrestricted angle is presented. In this scenario, the power converter operating region is represented by a circle, centered at the end of the LFT apparant power vector, as shown in Figure 13a. When utilized for voltage regulation, the power converter is able to inject a series voltage with a nonfixed phase angle, as shown in Figure 13b. In this case, the power converter voltage vectors are inside a circle centered at the end of the transformer winding voltage. Then, the load voltage corresponds to the summation of the series and transformer winding voltage vector. The additional degree of freedom when 
injecting the voltage vector allows for improved regulation capabilities provided by the converter, especially in a transient state.

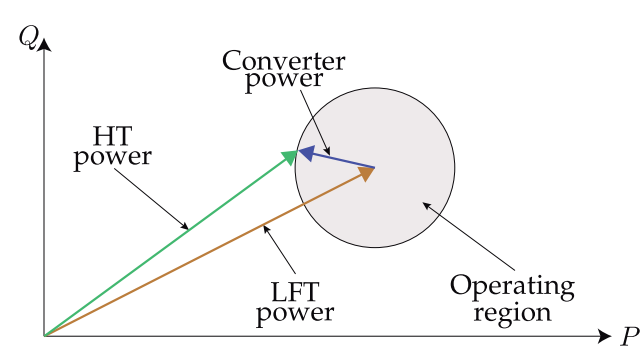

(a)

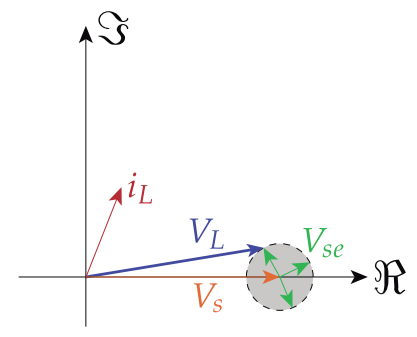

(b)

Figure 13. Operating region of HDTs whose power converters are capable of injecting active and reactive power. (a) PQ operating region. (b) Complex plane operating region for voltage compensation.

HDTs configurations based on AC/AC power converters, such as configurations of Figure $2 \mathrm{e}-\mathrm{j}$ are capable of delivering unrestricted active and reactive power only if the suitable power converters are utilized. For this purpose, power converters based on DCLink are required to provide independent active and reactive power. These topologies are presented in Figure 9. Additionally, the three-phase direct matrix converter of Figure 8 is capable of synthesizing an output voltage with varying phase by means of commutating the input phases. In the case of SST-based power converters, the topologies of Figure 10c,d are capable of operating in this region. In both cases the DABs establish the DC-Link voltage and then, by means of DC/AC converters, the desired output voltage is synthesized.

\subsubsection{Use of Tap Changers Systems to Extend the HDT Operating Region}

The voltage compensating range of configurations presented in this paper can be extended by means of the LFT taps changers. In the specific case of the configuration of Figure 2e, an alternative with tap changers is shown in Figure 14a. Tap changers commutation systems allow us to add an offset into the voltage regulation in steps, without an increase in the power converter rating. The operating region of this alternative is shown in Figure 14b $[96,101]$. When utilizing HDT configurations that are able to inject unrestricted active and reactive power, the operating vector can be represented, as shown in Figure 14c. In this scenario, when the vectors are synchronized with the transformer voltage, the power converter operating region is shifted along the real axis according to the taps changer state. The taps can be designed in order to have a continuous operating region, by means of superimposing the operating circles. Nonetheless, the use of taps transformers decreases the reliability of the HDT, as one of the main reasons for LFT failure is related to the malfunctioning of tap changers systems.

\subsection{Power Converter Location Effects}

The power converters location has an important role in the protection of the LFT, as they can prevent the load and grid voltage harmonics propagation.

\subsubsection{Shunt Converter Location}

Power converters connected in shunt configurations are able to provide current-based mitigation, such as power factor correction, harmonics filtering, neutral current mitigation, among others.

Disregarding the connection method utilized to integrate the shunt converter to the grid, it can be placed into the primary-side, secondary-side or into an auxiliary circuit, as shown in Figure 15. When the shunt converter is placed on the primary-side, as seen in Figure 15a, the current mitigation is made on the primary-side, being able to improve the grid power quality. On the other hand, in the presence of loads that generate harmonics currents, these are not mitigated on the secondary-side and, therefore, they are free to flow through the LFT. The presence of current harmonics on the LFT is harmful for the LFT 


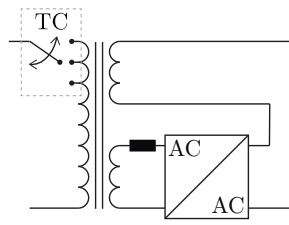

(a)

lifetime, due to the fact that they generate additional losses, acoustic noise, and vibrations, among others. The winding temperature, specifically its hot spot, is a critical variable for LFT as it has a critical impact on the winding isolation health. Therefore, as no mitigation system is placed on the secondary-side, the LFT lifetime decreases. One method to deal with harmonic currents on the LFT is transformer derating. Alternatively, if the shunt converter is placed on the secondary-side, as shown in Figure 15b, load harmonics can be compensated and prevent their circulation on the LFT and improving the LFT lifetime. Additionally, the connection of the shunt converter on the secondary-side can be achieved with simple power converter topologies, without requiring multilevel alternatives or CTs.

When the power converter is connected to an auxiliary winding, it can be utilized to provide current compensation by means of flux compensation. Under this scenario, the effects of the load currents on the primary-side current are mitigated. Although the transformer magnetic flux is compensated, load harmonic currents can circulate through the secondary-side winding. In order to compensate for those currents, the power converter must inject the required compensating harmonics currents into the auxiliary-winding, as represented in Figure 15c. The compensating currents depends on the load characteristic, the secondary and auxiliary winding ratios, and the auxiliary winding configurations. While the primary side winding currents are compensated in terms of power quality, the currents circulating through the secondary and auxiliary winding can contain high amounts of harmonics currents. Therefore, the LFT must be designed in order to operate under these conditions.

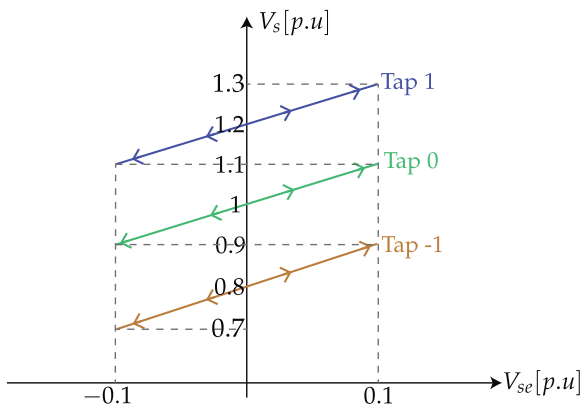

(b)

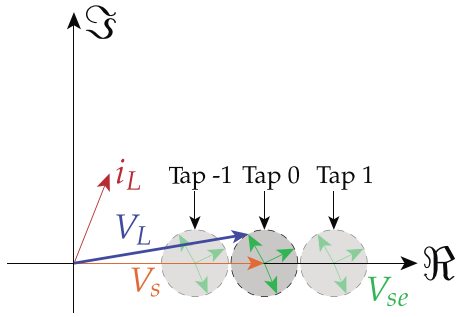

(c)

Figure 14. HDT with taps changers. (a) Diagram of a HDT configuration with taps changers systems (b) Operating region of the HDT output voltage versus the power converter converter output voltage (c) Complex plane operating region.

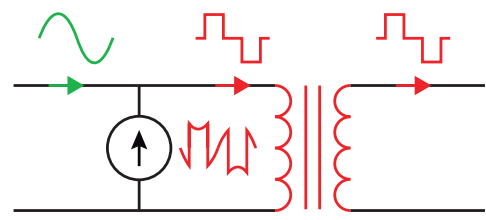

(a)

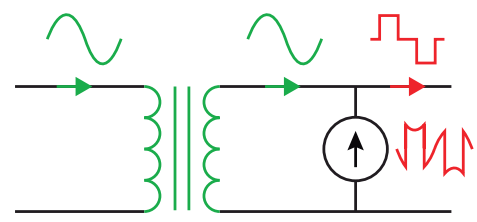

(b)

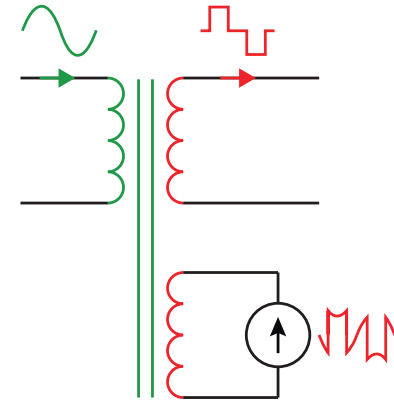

(c)

Figure 15. Shunt converter locations. (a) Primary-side. (b) Secondary-side. (c) Connected to an auxiliary winding.

\subsubsection{Series Converter Location}

Power converters connected in series to the distribution line are capable of mitigating voltage-based power quality problems, such as grid voltage sags, swells, voltage unbalances, and harmonics mitigation, among others. 
As with a shunt converter, the location of the series converter has a big impact on the LFT. When the series converter is placed on the primary-side, as in Figure 16a, the supplied voltage can be improved in terms of power quality, improving the LFT performance. The primary-side power converter can be utilized to actively regulate the load voltage. The same effect can be obtained if the converter is placed on the secondary-side, as in Figure 16b. Nonetheless, the LFT is unprotected from voltage disturbances, which can cause additional losses, inrush currents and malfunctioning on the rest of the grid.

An alternative to previous connection methods consists of connecting the power converter to an auxiliary winding, as in Figure 16c. In order to provide voltage regulation, the core to which the winding is attached, must be designed in a shunt configuration. The harmonic voltages coming from the grid and those injected by the power converter will affect the magnetic core paths associated with their windings. The resultant magnetic flux circulating in the magnetic path associated to the secondary-side winding will be improved in terms of power quality. Therefore, the resultant voltage waveform on the output converter terminal is improved. The magnetic core path associated with the primary and auxiliary-winding will be under the stress of disturbed voltage, causing additional core losses. Specially, considering nonlinear reluctance, voltage disturbances can cause inrush currents on both circuits.

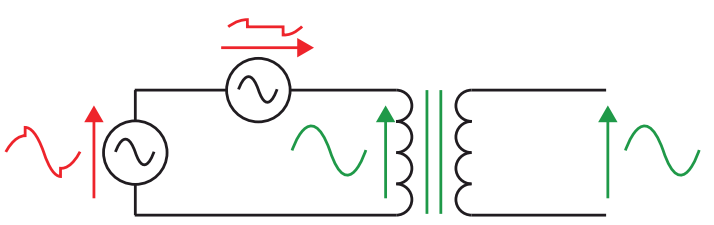

(a)

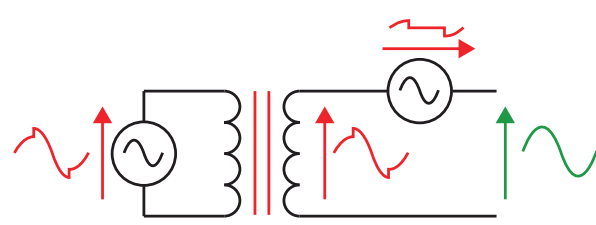

(b)

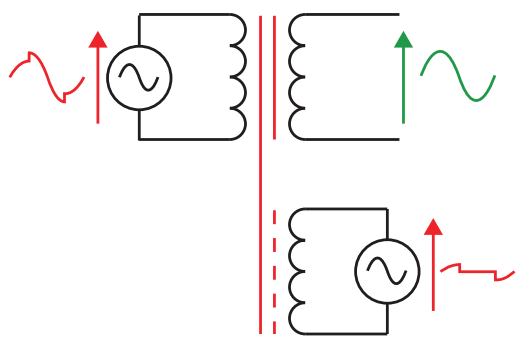

(c)

Figure 16. Series converter locations. (a) Primary-side. (b) Secondary-side. (c) Connected to an auxiliary winding.

\subsubsection{Combined Compensation and Circulating Active Power Flow}

It is possible to combine the series and shunt converters into one solution, as shown in the configurations of Figure 2e-i. Among these configurations, two groups can be highlighted. Configurations of Figure $2 \mathrm{e}, \mathrm{f}$ take their energy from the auxiliary winding and inject them in series to the distribution line. On the other hand, configurations of Figure $2 h, i$ take their energy from the secondary-side winding and inject them in series to the grid. In both groups, two scenarios can be analyzed according to the location of the series converter, i.e., if the series converter is connected to the primary or secondaryside. To carry out this analysis, and in order to simplify it, the following assumptions are considered: (1) The power converters and LFT are ideal. (2) The load is resistive, i.e., only active power is consumed. (3) The series converter is controlled to ensure that the rated voltage is provided to the primary-side winding. If its connected to the secondary-side, the series converter is controlled to ensure rated voltage to the load (4) The system operates under a voltage sag of $k[$ p.u.].

As shown in Figure 17a, when the series converter is placed on the secondary-side and the configuration of Figure $2 i$ is utilized, the load consumes a rated active power equals to $P_{L}$, as it is supplied with rated voltage. In order to compensate the voltage sag, 
the series converter must inject a series voltage in phase with the secondary-side voltage with magnitude equal to $k$ [p.u.]. As the rated current is flowing on the secondary-side, the power injected by the series unit is proportional to the voltage sag value, and it is equal to $k P_{L}$. The shunt converter acts as a support unit, and it is in charge of delivering this energy to the series converter. Thus, it consumes $k P_{L}$ from the secondary-side of the LFT. Consequently, the total amount of power delivered by the LFT is equal to the load, $P_{L}$. The power converter regulates the load voltage, and from the point of view of the LFT, the load and the power converter is seen as a constant power source.

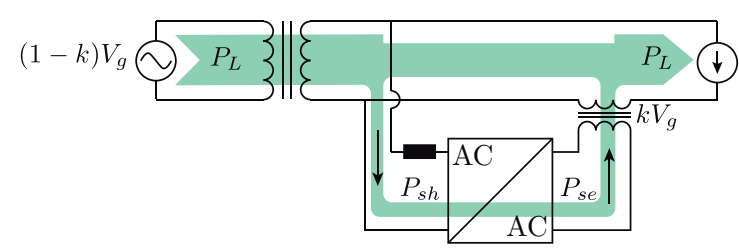

(a)

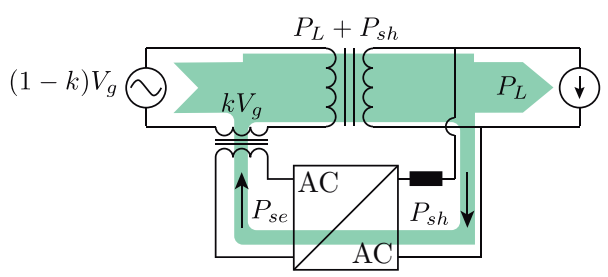

(b)

Figure 17. Circulating active power flow scenarios. (a) No circulating power between the LFT and the power converter. (b) Circulating power flow between the LFT and power converter.

When the CT is connected on the primary-side, as in Figure 17b, a feedback phenomena exist. The series converter injects a voltage equal to $k[p . u$.] in phase with the grid voltage. Due to the lossless LFT, rated load voltage is supplied, consuming rated active power, $P_{L}$. Thus, the series converters injects an active power equal to $P_{s e}$, which must be supplied by the shunt converter on the secondary-side. Therefore, the total power supplied by the LFT is equal to the sum of the rated load and the shunt converter active power, $P_{L}+P_{s h}$. As the primary-side winding voltage is controlled at its rated value, the current is proportional to $P_{L}+P_{s h}$ and it is higher than its rated value. Thus, when the power converter injects a voltage equal to $k\left[\right.$ p.u.], the required active power higher than $k P_{L}$, and it is given by the following equation.

$$
P_{s e}=P_{s h}=\frac{k}{1-k} P_{L}
$$

The circulating active power flow between the LFT and the power converter exists in the presence of a voltage disturbance, and in periods of high load, the additional amount of power can overload the LFT, affecting its lifetime. Under this scenario, the amount of active power processed by the LFT is equal to the load and the circulating active, and it is given by the next equation.

$$
P_{L F T}=\frac{1}{1-k} P_{L}
$$

Figure 18 presents the required injected active power by the power converter in order to compensate for different grid voltage sags, as well as the total amount of active power processed by the LFT. These values are calculated assuming that the power converter and the LFT are ideal. As is expected, the additional power requirements increases as the voltage sags to compensate are higher. If the power converter losses are considered, even higher values of active power will be required in order to compensate voltage sags, which decreases the overall efficiency. Additionally, if the LFT losses and leakage impedances are considered, the additional amount of circulating active power required generates additional voltage drops which must be compensated by the power converter. Therefore, the power converter must compensate for the grid voltage sag and the voltage drop generated by its operation, which can be considered as a higher equivalent voltage sag, increasing the required circulating active power flow and losses. 


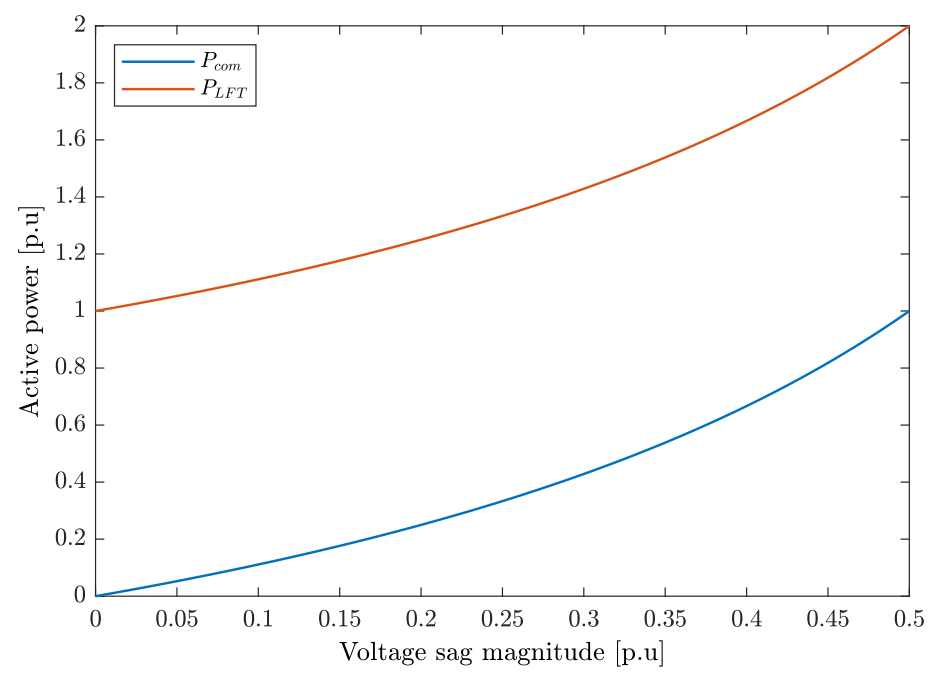

Figure 18. Required circulating active flow values to compensate voltage sags.

The circulating active power issue can be solved if the series power converter takes its energy from a DC source, such as a microgrid [66]. The energy obtained from the DC source breaks the active power loop between the transformer and the LFT, allowing to preserve the HDT configuration. Alternatively, the energy can be supplied from the primary-side by means of a shunt converter and then inject it in series to the medium voltage distribution line, breaking the circulating active power flow. This solution is presented in the combined configuration of Figure 3 [69]. The additional shunt converter in the secondary-side is used for load current compensation purposes. The integration of the primary-side shunt converter to the medium-voltage grid can be realized by means of CTs or by means of multilevel topologies. The latter is preferred in order to avoid and reduce the weight and volume of the CTs. By doing this, for a $k$ [p.u.] voltage sag, the required active power for voltage regulation is proportional to $k$. Additionally, independently of the voltage sag magnitude, the active power processed by the LFT is equal to the load.

\subsection{Losses in Distribution Transformers}

Transformer losses are composed of load and no-load losses. Load losses depend on the circulating currents generated by the loading of the distribution transformer. The main components are the heat losses in the transformer winding, which are generated by the $I^{2} R$ term. Stray losses belong to load losses, and they are subdivided as follows: (1) Winding stray losses: losses generated by the effect of leakage electromagnetic flux in the winding. (2) Other stray losses: losses generated by electromagnetic flux in the core, core clamps, magnetic shields, tank walls, bolts, among others [8].

No-load losses are those generated as a product of the transformer energization to establish the magnetic flux, without loads connected to the transformer winding. No-load losses are composed of the following terms: (1) Hysteresis losses in the core laminations. (2) Eddy current losses in the core laminations. (3) $I^{2} R$ losses due to the magnetizing current. (4) Dielectric losses [8]. The most relevant are hysteresis and eddy current losses, as they represent almost $99 \%$ of the no-load losses. Heat losses due to the magnetizing current are neglected, as magnetizing currents are a small fraction of the rated current of the transformer. In distribution networks applications dielectric losses are neglected as well, as these are relevant when the operating voltages are over $50 \mathrm{kV}$ [102]. The Steinmetz equation is utilized to represent the total core losses under sinusoidal input voltages [103].

$$
P_{f e}=K_{c} f^{\alpha} B_{\max }^{\beta}
$$

To cope with the variability of the loads, distribution transformers are designed to operate with maximum efficiency with loading between $40 \%$ and $60 \%$. Above that, the efficiency starts to decrease due to the winding losses. On the other hand, under low 
loading operating, core losses are predominant, leading to a poor and unsustainable operation [104]. For example, for a $1000 \mathrm{kVA}$ distribution transformer operating with unit power factor, the efficiency at $10 \% 45 \%$ and $100 \%$ load are $96.99 \%, 98.70 \%$, and $98.37 \%$, respectively. The efficiency is worsened under different grid conditions, such as low power factor [105]. In light-load scenarios, the power converters of the HDT can be utilized to improve the efficiency [106].

The effect of the power converter location in terms of the presence of harmonic currents and voltages in the distribution transformer was shown in a previous section. The effect of harmonics in the losses of the transformer and its lifetime will be summarized, which is a handful in order to evaluate the protection level given to the distribution transformer by each HDT configuration. Generally speaking, grid voltage harmonics affect the magnetic core losses, while load harmonics affect the winding and structure losses [107].

The applied voltage magnitude has a critical impact on the insulation materials of the transformer, as high $\mathrm{dv} / \mathrm{dt}$ can create high electric fields. In the presence of distorted grid voltages, the $\mathrm{dv} / \mathrm{dt}$ increases, accelerating the degradation of the insulation materials and reducing the transformer lifetime [107]. In [102], different nonlinear voltage profiles have been studied and it is shown that they have a drastic impact on the transformer no-load losses, and increase the maximum flux density of the magnetic core. Furthermore, the magnetic flux inside the core is nonuniform, which generates partial saturation, temperature rise, among other issues. In [9], a distribution transformer is tested under different operating regimes. In the case of a line-to-line voltage THD of about $12 \%$, the no-load losses and no-load current increase in about $3.46 \%$ and $37.3 \%$ compared to a sinusoidal voltage. The work in [10] analyzes the effect of grid voltage harmonics on the distribution transformer performance. Some results show that the core losses increase by about $21 \%$ when the voltage harmonics are given by the worst-case scenario of the harmonics limits given by IEC 61000-3-6. Additionally, the results of the impact of third and fifth harmonics on the temperature of the transformer are presented. Compared to a pure sinusoidal voltage, there is an $8.2 \%$ and $11.8 \%$ temperature increase of the hottest spot, for $3 \%$ of third and fifth harmonics. The system presents higher losses as the frequency increases due to the effect of temperature and skin effect on the winding resistance.

Compared to voltage harmonics, load harmonics have a bigger impact on the transformer losses and lifetime [108]. The study of load harmonics and the effect on the transformer is crucial for the transformer design, as it gives guidance for its derating. The IEEE std C57.110 establish recommended practices for liquid-filled and dry-type transformer when supplying non-linear loads. Among the load losses, winding stray losses depend on the square of the frequency, while the exponent of other stray losses is less than one. When a transformer operates supplying nonlinear currents, the additional winding stray losses increase the hot-spot temperature [8]. The rise in the temperature affects the insulation systems, reducing the transformer lifetime. The hot-spot temperature $\left(\theta_{H}\right)$, can be utilized to calculate the aging acceleration factor $\left(F_{A A}\right)$, which, for a mineral-oil immersed transformer, is given as follows [109].

$$
\begin{gathered}
\theta_{H}=\theta_{A}+\Delta \theta_{T o}+\Delta \theta_{H} \\
F_{A A}=e^{\frac{15000}{110+273}-\frac{15000}{\theta_{H}+273}}
\end{gathered}
$$

where $\theta_{A}$ is the ambient temperature, $\Delta \theta_{T o}$ is the oil temperature rise over the ambient temperature, and $\Delta \theta_{H}$ is the winding hot-spot temperature rise over the top oil temperature.

In [110] a load characterized by consuming high fifth and seventh harmonics is analyzed utilizing three different methods to calculate the transformer losses and estimated lifetime. Under the analyzed operating conditions, the minimum $F_{A A}$ is equal to 1.12 , which for a transformer with an expected operating time of 30 years, the lifetime is decreased in a $10 \%$. In [111], a load current profile given by $5 \%, 6 \%$, and $5 \%$ for the third, fifth and seventh harmonic is tested. Under these operating conditions, the core losses increase by about $15.5 \%$, with respect to a full sinusoidal case [111]. 
The impact of nonlinear voltages and currents on the distribution transformer can not be neglected. Therefore, the power converter unit of the HDT can be utilized to damp the impact of harmonics on the transformer lifetime. Nonetheless, it will depend on the utilized HDT configuration.

\subsection{LFT Protection}

Based on the HDT configurations, the operating region of the HDT, the location of the power converters, and the circulating active power issue, the protection level provided to the LFT can vary. In order to evaluate the protection level, Table 4 summarizes the assessment points considered. The LFT is protected from grid voltage disturbances when the series power converter is located in the primary-side and it is able to provide active and reactive power for voltage mitigation. In this case, the protection is assessed with 3 points. When the power converter is only capable of providing reactive power, such as in the case of self-supported configurations, the system is assessed with 2 points. In a similar manner, when the shunt converter is located in the secondary-side and it is capable of providing active and reactive power, the protection is assessed with 3 points. Otherwise, if only reactive power is delivered, 2 points are given. In both cases, series and shunt compensation, voltage and currents harmonics are diminished, therefore improving the operating conditions of the LFT. On the other hand, when magnetic compensation is utilized, the system is evaluated with 2 and 1 point, for active plus reactive and reactive power compensation, respectively. In this case the assigned score is less due to voltage and currents harmonics can circulate through the LFT windings, which affects the winding insulation. Additionally, when circulating active power flows occurs, the system is evaluated with -1 point. Only the basic configurations are assessed in this work, and alternatives such as those that use taps changers or shunt converters connected to the LFT taps are not evaluated. Then, for each HDT configuration of Figure 2, the protection provided the LFT is assessed and summarized in Table 5 according to the the presence and location of the series converter. In the presence of shunts converter, it is assumed that they are always connected on the secondary-side in order to provide load current compensation.

Table 4. LFT protection assessment.

\begin{tabular}{cc}
\hline Motive & Assessment \\
\hline Circulating active power flow & -1 \\
\hline Magnetic compensation $(\mathrm{Q})$ & 1 \\
\hline Magnetic compensation $(\mathrm{P}+\mathrm{Q})$ & 2 \\
\hline Series converter on primary-side $(\mathrm{Q})$ & 2 \\
\hline Shunt converter on secondary-side $(\mathrm{Q})$ & 2 \\
\hline Series converter on primary-side $(\mathrm{P}+\mathrm{Q})$ & 3 \\
\hline Shunt converter on secondary-side $(\mathrm{P}+\mathrm{Q})$ & 3 \\
\hline
\end{tabular}


Table 5. Protection provided to the LFT.

\begin{tabular}{|c|c|c|c|c|c|}
\hline & & \multicolumn{4}{|c|}{ LFT Protection } \\
\hline & & No Ser. & Ser. sec. & Ser. pri. & Ser. mag. \\
\hline \multirow{4}{*}{ 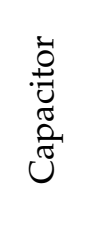 } & (a) & - & 0 & 2 & - \\
\hline & (b) & - & 0 & 2 & - \\
\hline & (c) & 1 & - & - & - \\
\hline & (d) & 2 & - & - & - \\
\hline \multirow{3}{*}{ 宏若 } & (e) & - & 1 & 3 & - \\
\hline & $(\mathrm{f})$ & - & 1 & 3 & - \\
\hline & $(g)$ & - & - & - & 3 \\
\hline \multirow{3}{*}{ ن } & (h) & - & 2 & 4 & - \\
\hline & (i) & - & 2 & 4 & - \\
\hline & (j) & 3 & - & - & - \\
\hline
\end{tabular}

\subsubsection{Self-Supported HDT}

For the series configuration of Figure 2a, no protection is provided to the LFT, since the power converter is located on the secondary-side. Therefore, the grid voltage harmonic can affect LFT operation. Alternatively, it can be connected on the primary-side by means of a $\mathrm{CT}$, as shown in configuration Figure $2 \mathrm{~b}$. Assuming an operation without DC sources, the level of protection is assessed with 2 points, due to that the only reactive power is utilized for the compensation, being unable to provide full voltage protection.

For the shunt configuration of Figure 2d, the maximum level of protection to the LFT occurs when the converter is connected to the secondary-side. In this case, it is assessed with 2 points, due to the use of reactive power, exclusively.

When the power converter is connected to the auxiliary winding in order to provide flux compensation, as in Figure 2c, load harmonics and the current injected by the power converter can circulate through the secondary and auxiliary windings, negatively impacting on the LFT lifetime. The protection level is lower compared to when the converter is connected to the secondary-side. The power converter is able to inject reactive power; therefore, this configuration is assessed with 1 point.

\subsubsection{HDT Connected to Auxiliary Windings}

For the configuration of Figure 2e, due to the fact that the location of the series converter is on the secondary-side, no voltage protection is provided. On the other hand, if the power converter can control its input current for flux compensation, only reactive power can be utilized for this task. Therefore, the protection is assessed with 1 point. Alternatively, if the connection is made on the primary-side, for example, connecting the converter to the ground-side of the primary winding, a circulating active power flow is generated. Therefore, the configuration is assessed with 3 points in this case. The same occurs for configuration of Figure 2f.

For configuration of Figure 2g, the harmonics currents of the load and the shunt converter can circulate through the LFT winding. On the other hand, grid voltage disturbances can affect the LFT core, as well as the compensating voltages injected by the series converter. Therefore, this configuration is assessed with 3 points.

\subsubsection{HDT Connected to the LFT Primary or Secondary Windings}

Regarding the protection level provided by configuration of Figure $2 \mathrm{~h}$, no voltage protection is provided when the series converter is placed on the secondary-side. On the other hand, the shunt converter can be utilized to compensate load harmonics currents. Therefore this configuration is assessed with 2 points. Alternatively, if the series power 
converter is placed on the primary-side, it can provide full voltage regulation. Nonetheless, circulating active power flows occurs. Therefore, in this case, this configuration is assessed with 4 points. The same scenario can be expected for configuration of Figure 2i.

In configuration of Figure $2 \mathrm{j}$, no direct voltage regulation is provided, due to both ends of the power converter are connected in shunt configuration. Nonetheless, a certain degree of voltage regulation can be provided by means of reactive power injection, it is not considered for the assessment. Therefore, this configuration is evaluated with 3 points, since full current control can be provided. Alternatively, this configuration can be utilized in periods of high energy demand, and utilize the power converter in order to alleviate the LFT.

\subsection{HDT Reliability}

Distribution transformers are highly reliable elements, being able to operate under faults, for example delivering high fault currents for several seconds. When integrating power electronics into the distribution transformers, it is expected that this desirable characteristic is maintained.

Each configuration and topology must be considered in the design of the HDT to provide a reliable operation; nonetheless, not enough research has been carried out in this area. In general, for HDTs with series-connected converters, it is considered a bypass switch that disconnects the power converter in the case of faults $[25,49]$. For the configuration of Figure 19, a comprehensive protection system design have been realized [26,27]. In the case of short circuits on the low-voltage grid, high currents are delivered by the transformer and the power converter, which can damage the latter. Therefore, in this scenario, the power converter must be bypassed rapidly, allowing the transformer to deliver the short circuit currents. A bidirectional switch composed of antiparallel high current thyristors is utilized, which provides a low-impedance path in less than $100 \mu$ s. Additionally, the reliable design of the HDT considers diverse scenarios, such as lightning surges and medium voltage surges, for which it is necessary to find a compromise between the auxiliary winding ratio, power converter losses, semiconductor breakdown voltage, and filter size, among others.

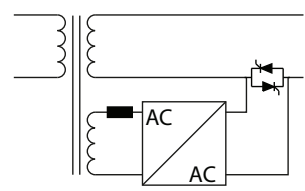

Figure 19. Hybrid transformer of configuration Figure 2e with bypass switch.

A reliable HDT must be able to detect a dangerous operating condition and depending on the configuration, bypass and/or disconnect the power converter from the grid and transformer [49]. When done correctly, the HDT behaves as a conventional LFT, ensuring high reliability. Alternatively, reliability can be assessed according to the power converter topology and its under-fault operation capability. For example, for two-level converters, redundant legs can be included which can be switched on in the case of faults. Additional fault isolation circuits are mandatory to isolate the damaged leg as fast as possible [112]. On the other hand, for configurations connected to the primary-side, the use of mediumvoltage multilevel converters adds a level of reliability compared to, for example, two-level topologies. When a submodule of a cascaded multilevel converter fails, the system can be reconfigured to continue with its normal operation, without requiring one to disconnect the power converter [113].

\section{Ancillary Services Provided by Hybrid Transformers}

In conventional electrical market structures, ancillary services are provided by the synchronous generators to satisfy the power system requirements at the transmission level, which typically considers frequency, voltage, and reactive power regulation. These services are traded in the ancillary services market, where the transmission system operator 
is the purchaser [114]. On the other hand, the increase in distributed renewable energy resources and the inclusion of power electronics in distribution systems has brought new challenges into the operation of distribution networks but has also broadened the spectrum of ancillary services that can be provided. In this regard, the power converter of the HDT can be utilized to provide ancillary services.

In terms of cost, an HDT is expected to have a higher price compared to a conventional LFT due to the inclusion of the power converter. Some aspects that will determine the cost of an HDT are the number of power converters taken into consideration, i.e., the number of series and/or shunt converters, and the power converter topology. The converter location plays an important role in the costs-as the integration of the power converter into the medium-voltage is more technically challenging than its low-voltage counterpartrequiring, for example, the use of multilevel power converter or isolation transformers. Beyond the cost of the power converter, the additional services that can be provided by the HDT can make it an interesting alternative for electrical companies, as the HDT services can be part of the ancillary services market.

Besides the previously mentioned capabilities of an HDT, such as current and voltage harmonic filtering, power factor correction, voltage regulation, load balancing, neutral current mitigation, among others, other applications of HDTs have been researched. The provided ancillary services types and their impact on the grid or distribution transformer will depend on the selected HDT configuration and topology. Some of these services are presented in the following subsections.

\subsection{Distribution Transformer Inrush Current Mitigation}

There are several methods to reduce the impact of inrush currents at the moment of the distribution transformer connection. For example, connecting the transformer phases in sequences at optimal moments. Nonetheless, it is possible to use the shunt stage of HDTs to mitigate their impact. In this scenario and based on Figure 20, using a prefluxing technique, in which the converter induces a magnetic flux equal to that induced by the grid at the connection instant, reducing the impact on the inrush currents generation. Once the transformer is connected to the grid, the power converter can be synchronized to the secondary side to initiate its normal operation, regulating the secondary side voltages and currents. When it is desired to disconnect the transformer, it is possible to use the same technique in the reverse order until the transformer flux is zero. This feature is proposed utilizing the configuration of Figure 2e along with a DC storage system connected to the DC-Link to provide the necessary energy to magnetize the transformer [115].

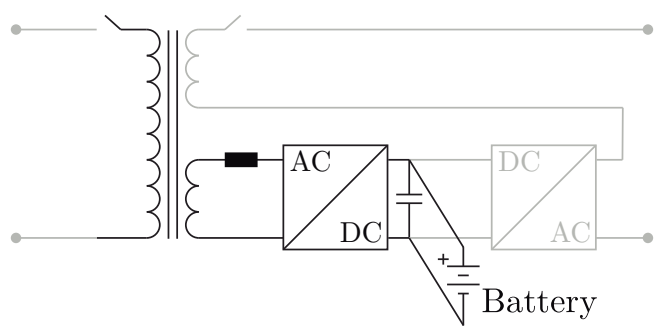

Figure 20. HDT configuration for inrush current mitigation.

Alternatively, the same configuration can be applied to reduce magnetizing flux DC offsets, which generate inrush currents. The mitigation is carried out by the power converter module connected to the auxiliary winding, modifying the core flux [116].

\subsection{Distribution Transformer Additional Capacity}

By means of a parallel connection of an SST, such as configuration of Figure $2 \mathrm{j}$, and by utilizing active and reactive power control, it is possible to increase the capacity of an LFT. This scenario is represented in Figure 21. The power control is designed to supply a desired load proportion, while being able to provide power quality problem mitigation 
services. This solution is carried out without a considerable volume or complexity increase. Proposed solutions integrate a full rated power converter in parallel to the conventional transformer $[68,117,118]$.

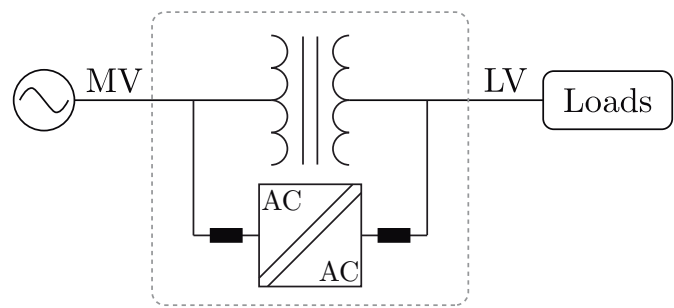

Figure 21. Increase in an LFT capacity by means of a HDT.

\subsection{Hybrid Transformers to Provide Virtual Inertia}

In DC-Link configuration-based HDTs, it is possible to employ DC storage systems or distributed generation systems, which can be used to provide grid frequency support. This is highly attractive in low-mechanical inertia grids, where the use of HDTs can be exploited [66]. Additionally, if multiple units are employed in a power system, they can have an important effect even in stiff grids. Figure 22 shows the DC system integration to the HDT DC-Link to provide grid frequency support.

By means of an external control loop, it is possible to improve the grid inertia, which allows us to soften the frequency dynamic behavior under contingencies, providing primary frequency support. The control is based on modifying the converter injected power according to the grid frequency variation, emulating the synchronous generator mechanical equation $[119,120]$.

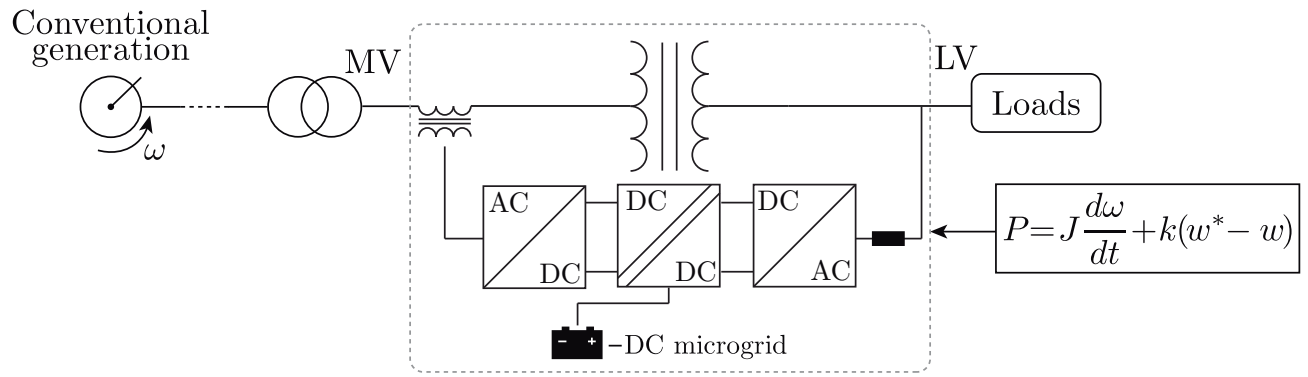

Figure 22. HDT with virtual inertia.

\subsection{Renewable Energy Systems and New Kind of Loads Integration}

Photovoltaic or wind generation systems can be integrated into the grid through conventional transformers. Their electromagnetic characteristic has a high impact on power quality, especially during low radiation and low wind speed periods. This operating scenario is translated into low injected active power by the renewable generating units and makes the magnetizing transformer currents considerable with respect to load currents. The generated harmonics by this phenomenon mainly depend on the winding connection type and the harmonic content presented on the grid voltage.

Figure 23a shows the presented scenario employing a HDT, which uses the configuration of Figure 2j. It allows for the attenuation of the renewable systems impact on the power electric system through an appropriate control system, which accounts for the transformer currents into the model. By doing this, the grid current on the medium voltage side is proportional to the current injected by the renewable generating units for all radiation and wind speed range. Additionally, it is possible to design the controllers to operate with unit power factor if the converter rated power allows it [67].

New kind of loads, such as electric vehicles, can produce additional stress on distribution transformers, for instance during multiple charging processes occurring at evening and night-time, specially at the domiciliary level [16]. The use of a HDT, such as exemplified in 
Figure 23b, can be beneficial for the HDT lifetime, as the power converter can be used to dampen the power consumption and also avoid LFT overloading.

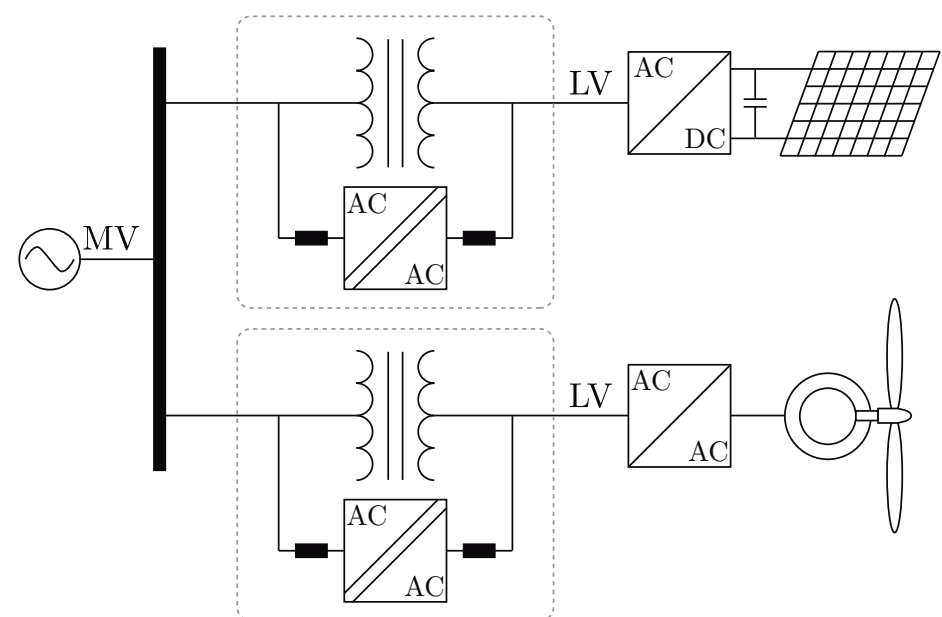

(a)

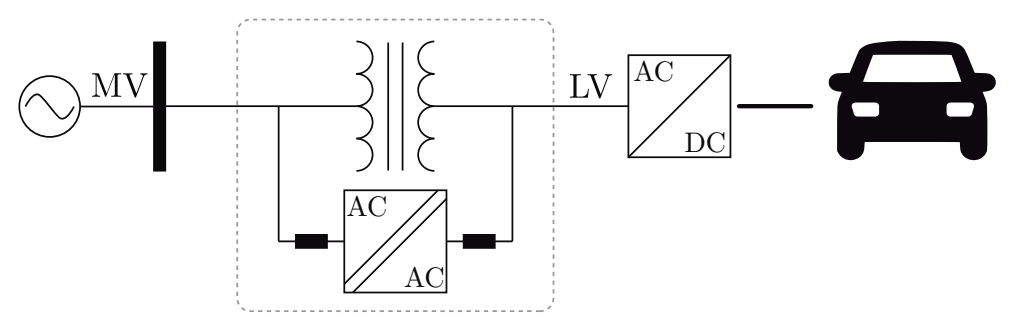

(b)

Figure 23. HDTs for the integration of renewable systems and new kind of loads. (a) Renewable systems integration. (b) Electric vehicle charging station.

\subsection{Decentralized Control of a HDT for Voltage Regulation in Active Networks}

The expansion of distributed generation on the grid adds several challenges into the distribution network planning. One of the main challenges is the effect of the intermittence of the renewable sources, which can have a big impact on the voltage profile of the distribution network. Therefore, the use of smart devices and control techniques are required in order to cope with these challenges.

Active distribution network can take advantage of HDTs in order to improve the voltage profiles. For example, the HDT configuration of Figure 2e can be utilized to provide voltage control to the low-voltage side by means of the series converter. On the other hand, the power converter connected to the auxiliary winding can absorb/inject a limited amount of reactive power which, in conjunction with other HDTs or smart devices, can provide voltage regulation to the medium-voltage buses.

Multiple HDTs, as shown in Figure 24, can be coordinated in order to regulate the voltage magnitude of the medium-voltage buses, while optimizing the reactive power injection/consumption of the HDTs. This task can be done in a decentralized manner, utilizing HDTs controlled with local variables, exclusively [121]. 


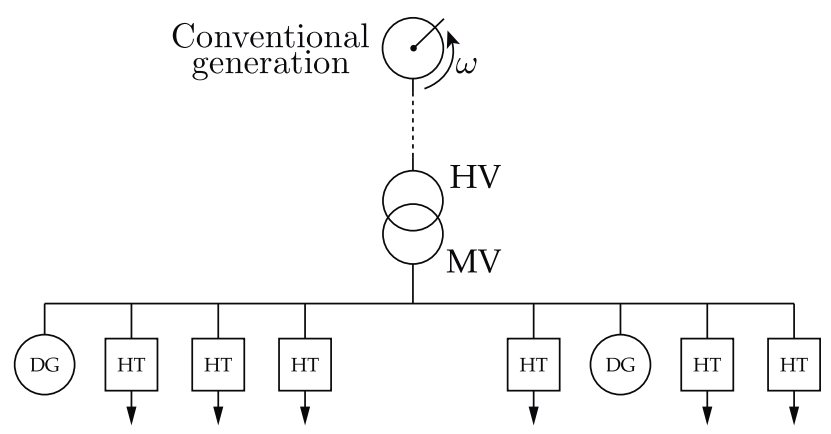

Figure 24. Active distribution grid voltage regulation by means of HDTs.

\section{Conclusions}

Nowadays, new kinds of loads are connected to the distribution network by means of power converters that are supplied by conventional transformers. In the case of electric vehicles and renewable sources, such as wind and photovoltaic system, their highly variable penetration increases the stress on the distribution transformers. Additionally, the presence of harmonics, due to the operation of the power converters worsens their operating conditions.

HDTs are an attractive solution to tackle down several power quality problems. Multiple HDT configurations exist, depending on where the power converters obtain their energy and how they inject it back to the grid. The use of each configuration will depend on the system purpose, i.e., to provide load support, grid support, LFT support, or combinations of previous objectives. Additionally, the ability to retrofit is an important aspect in the process of the HDT design. Configurations based on AWs will require an LFT specially designed for the operating conditions, where the LFT windings will suffer the effects of nonlinear currents. On the other hand, solutions that aim for retrofitting will require the use of CTs or HFTs in order to provide the required isolation as it is not possible to connect the power converter on the ground-side of the LFT where, typically, the neutral connection is made in the LFT.

Nonetheless, it is still necessary continue studies on the different configurations and topologies available, as main research are focused on a small number of them. Up until now, most studies are focused on the modeling and control of HDTs. It is necessary to carry out research on the different HDT configuration and topologies, their protection systems and their compatibility with other distributed resources, and especially on complementary services.

As HDTs are based on power converter, they are able to provide several complementary services, such as LFT inrush current mitigation, DC sources integration, virtual inertia and medium-voltage grid voltage support. These complementary services contribute to the strengthening of the power system, which is subject to unpredictable disturbances, specially nowadays, when new kinds of loads are being connected to it.

Author Contributions: A.C. and M.P. wrote the original draft. C.B., A.H., S.R. and M.M. reviewed the manuscript. All authors have read and agreed to the published version of the manuscript.

Funding: This work was supported by the Fondecyt Project 1181839 and 1201308 , by the Advanced Center for Electrical and Electronics Engineering AC3E (CONICYT/FB0008), by SERC Chile (CONICYT/FONDAP/15110019), and by CONICYT-PFCHA/Doctorado Nacional/2019-21191478.

Institutional Review Board Statement: Not applicable.

Informed Consent Statement: Not applicable.

Data Availability Statement: Not applicable.

Conflicts of Interest: The authors declare no conflict of interest. 


\section{References}

1. Gönen, T. Electric Power Distribution System Engineering, 3rd ed.; CRC Press: Boca Raton, FL, USA, 2014.

2. Ciuriuc, A.; Dumitran, L.M.; Notingher, P.V.; Badicu, L.V.; Setnescu, R.; Setnescu, T. Lifetime Estimation of Vegetable and Mineral Oil Impregnated Paper for Power Transformers. In Proceedings of the 2016 IEEE International Conference on Dielectrics (ICD), Montpellier, France, 3-7 July 2016; Volume 2, pp. 720-723.

3. Fuchs, E.; Masoum, M. Power Quality in Power Systems and Electrical Machines, 2nd ed.; Academic Press: Cambridge, MA, USA, 2015.

4. IEEE. IEEE Recommended Practice for Monitoring Electric Power Quality, IEEE Std 1159-2019; IEEE: New York, NY, USA, 2019; pp. 1-98.

5. IEEE. IEEE Recommended Practice and Requirements for Harmonic Control in Electric Power Systems, IEEE Std 519-2014; IEEE: New York, NY, USA, 2014; pp. 1-29.

6. Singh, B.; Chandra, A.; Al-Haddad, K. Power Quality: Problems and Mitigation Techniques; Wiley: Hoboken, NJ, USA, 2015.

7. Sharma, A.; Rajpurohit, B.S.; Singh, S.N. A Review on Economics of Power Quality: Impact, Assessment and Mitigation. Renew. Sustain. Energy Rev. 2018, 88, 363-372. [CrossRef]

8. IEEE. IEEE Recommended Practice for Establishing Liquid Immersed and Dry-Type Power and Distribution Transformer Capability When Supplying Nonsinusoidal Load Currents, IEEE Std C57.110-2018; IEEE: New York, NY, USA, 2018; pp. 1-68.

9. Kefalas, T.D.; Kladas, A.G. Harmonic Impact on Distribution Transformer No-Load Loss. IEEE Trans. Ind. Electron. 2010, 57, 193-200. [CrossRef]

10. Dao, T.; Phung, B.T. Effects of Voltage Harmonic on Losses and Temperature Rise in Distribution Transformers. IET Gener. Transm. Distrib. 2018, 12, 347-354. [CrossRef]

11. Mazzanti, G.; Lusetti, L.; Fragiacomo, A. The State of the Art About Electric Arc Furnaces for Steel Use and the Compensation of Their Perturbing Effects on the Grid. In Proceedings of the International Symposium on Power Electronics Power Electronics, Electrical Drives, Automation and Motion, Sorrento, Italy, 20-22 June 2012; pp. 1277-1282.

12. Awadallah, M.A.; Venkatesh, B.; Singh, B.N. Impact of Solar Panels on Power Quality of Distribution Networks and Transformers. Can. J. Electr. Comput. Eng. 2015, 38, 45-51. [CrossRef]

13. Ustun, T.S.; Hashimoto, J.; Otani, K. Impact of Smart Inverters on Feeder Hosting Capacity of Distribution Networks. IEEE Access 2019, 7, 163526-163536. [CrossRef]

14. Kraiczy, M.; Stetz, T.; Braun, M. Parallel Operation of Transformers With on Load Tap Changer and Photovoltaic Systems With Reactive Power Control. IEEE Trans. Smart Grid 2018, 9, 6419-6428. [CrossRef]

15. Paterakis, N.G.; Pappi, I.N.; Erdinc, O.; Godina, R.; Rodrigues, E.M.G.; Catalao, J.P.S. Consideration of the Impacts of a Smart Neighborhood Load on Transformer Aging. IEEE Trans. Smart Grid 2016, 7, 2793-2802. [CrossRef]

16. Hilshey, A.D.; Hines, P.D.; Rezaei, P.; Dowds, J.R. Estimating the Impact of Electric Vehicle Smart Charging on Distribution Transformer Aging. IEEE Trans. Smart Grid 2013, 4, 905-913. [CrossRef]

17. Santos-Martin, D.; Lemon, S.; Watson, J.D.; Wood, A.R.; Miller, A.J.; Watson, N.R. Impact of Solar Photovoltaics on the Low-Voltage Distribution Network in New Zealand. IET Gener. Transm. Distrib. 2016, 10, 1-9.

18. Gong, Q.; Midlam-Mohler, S.; Marano, V.; Rizzoni, G. Study of PEV Charging on Residential Distribution Transformer Life. IEEE Trans. Smart Grid 2012, 3, 404-412. [CrossRef]

19. Faiz, J.; Siahkolah, B. Electronic Tap-Changer for Distribution Transformers; Springer: Berlin/Heidelberg, Germany, 2011.

20. Das, B.P.; Radakovic, Z. Is Transformer kVA Derating Always Required Under Harmonics? A Manufacturer's Perspective. IEEE Trans. Power Deliv. 2018, 33, 2693-2699. [CrossRef]

21. Abu-Siada, A.; Budiri, J.; Abdou, A. Solid State Transformers Topologies, Controllers, and Applications: State-of-the-Art Literature Review. Electronics 2018, 7, 298. [CrossRef]

22. Huber, J.E.; Kolar, J.W. Solid-State Transformers: On the Origins and Evolution of Key Concepts. IEEE Ind. Electron. Mag. 2016, 10, 19-28. [CrossRef]

23. Huang, A.; Zhu, Q.; Wang, L.; Zhang, L. $15 \mathrm{kV} \mathrm{SiC} \mathrm{MOSFET:} \mathrm{An} \mathrm{Enabling} \mathrm{Technology} \mathrm{for} \mathrm{Medium} \mathrm{Voltage} \mathrm{Solid} \mathrm{State}$ Transformers. CPSS Trans. Power Electron. Appl. 2017, 2, 118-130. [CrossRef]

24. Bala, S.; Das, D.; Aeloiza, E.; Maitra, A.; Rajagopalan, S. Hybrid Distribution Transformer: Concept Development and Field Demonstration. In Proceedings of the 2012 IEEE Energy Conversion Congress and Exposition (ECCE), Raleigh, NC, USA, 15-20 September 2012; pp. 4061-4068.

25. Burkard, J.; Biela, J. Hybrid Transformers for Power Quality Enhancements in Distribution Grids-Comparison to Alternative Concepts. In Proceedings of the NEIS 2018; Conference on Sustainable Energy Supply and Energy Storage Systems, Hamburg, Germany, 20-21 September 2018; pp. 1-6.

26. Burkard, J.; Biela, J. Design of a Protection Concept for a 100kVA Hybrid Transformer. IEEE Trans. Power Electron. 2019, 35, 3543-3557. [CrossRef]

27. Burkard, J.; Biela, J. Protection of Hybrid Transformers in the Distribution Grid. In Proceedings of the 2016 18th European Conference on Power Electronics and Applications (EPE'16 ECCE Europe), Karlsruhe, Germany, 5-9 September 2016; pp. 1-10.

28. Sastry, J.; Bala, S. Considerations for the Design of Power Electronic Modules for Hybrid Distribution Transformers. In Proceedings of the 2013 IEEE Energy Conversion Congress and Exposition, ECCE 2013, Denver, CO, USA, 15-19 September 2013; pp. 1422-1428. 
29. Huber, J.E.; Kolar, J.W. Applicability of Solid-State Transformers in Today's and Future Distribution Grids. IEEE Trans. Smart Grid 2019, 10, 317-326. [CrossRef]

30. Bala, S. Distribution Transformer. EU Patent PCT/US2013/056393, 23 August 2013.

31. Haj-Maharsi, M.Y.; Sandeep, B.; Tang, L. Hybrid Distribution Transformer with an Integrated Voltage Source Converter. US Patent US 201O/O220499 A1, 2 February 2010.

32. Haj-Maharsi, M.Y.; Tang, L.; Gutierrez, R.; Bala, S. Hybrid Distribution Transformer with AC \& DC Power Capabilities. US Patent US 2010/0201338 A1, 12 August 2010.

33. Haj-Maharsi, M.Y.; Tang, L.; Gutierrez, R.; Bala, S. Hybrid Distribution Transformer Having a Power Electronic Module for Controlling Input Power Factor and Output Voltage. US Patent US 9768704 B2, 19 September 2017.

34. Takahashi, Y.; Isobe, T.; Tadano, H. Series Reactive Power Compensator with Reduced Capacitance for Hybrid Transformer. In Proceedings of the 2018 International Power Electronics Conference (IPEC-Niigata 2018 -ECCE Asia), Niigata, Japan, 20-24 May 2018; pp. 3375-3382.

35. Omar, R.; Rahim, N.A. Voltage Unbalanced Compensation Using Dynamic Voltage Restorer Based on Supercapacitor. Int. J. Electr. Power Energy Syst. 2012, 43, 573-581. [CrossRef]

36. Newman, M.J.; Holmes, D.G.; Nielsen, J.G.; Blaabjerg, F. A Dynamic Voltage Restorer (DVR) With Selective Harmonic Compensation at Medium Voltage Level. IEEE Trans. Ind. Appl. 2005, 41, 1744-1753. [CrossRef]

37. Liu, Q.; Li, Y.; Liu, F.; Hu, S.; Xie, B.; Luo, L.; Cao, Y. A Controllably Inductive Power Filtering Method for Large-Power Industrial Rectifier System. In Proceedings of the 2016 IEEE 16th International Conference on Environment and Electrical Engineering (EEEIC), Florence, Italy, 7-10 June 2016.

38. Li, Y.; Peng, Y.; Liu, F.; Sidorov, D.; Panasetsky, D.; Liang, C.; Luo, L.; Cao, Y. A Controllably Inductive Filtering Method With Transformer-Integrated Linear Reactor for Power Quality Improvement of Shipboard Power System. IEEE Trans. Power Deliv. 2017, 32, 1817-1827. [CrossRef]

39. Li, Y.; Liu, Q.; Hu, S.; Liu, F.; Cao, Y.; Luo, L.; Rehtanz, C. A Virtual Impedance Comprehensive Control Strategy for the Controllably Inductive Power Filtering System. IEEE Trans. Power Electron. 2017, 32, 920-926. [CrossRef]

40. Sreenivasarao, D.; Agarwal, P.; Das, B. Neutral Current Compensation in Three-Phase, Four-Wire Systems: A Review. Electr. Power Syst. Res. 2012, 86, 170-180. [CrossRef]

41. Corasaniti, V.F.; Barbieri, M.B.; Arnera, P.L.; Valla, M.I. Hybrid Active Filter for Reactive and Harmonics Compensation in a Distribution Network. IEEE Trans. Ind. Electron. 2009, 56, 670-677. [CrossRef]

42. Zhang, L.; Sen, S.; Huang, A.Q. 7.2-kV/60-A Austin SuperMOS: An Intelligent Medium-Voltage SiC Power Switch. IEEE J. Emerg. Sel. Top. Power Electron. 2020, 8, 6-15. [CrossRef]

43. Zhu, Q.; Wang, L.; Chen, D.; Zhang, L.; Huang, A.Q. Design and Implementation of a 7.2 kV Single Stage AC-AC Solid State Transformer Based on Current Source Series Resonant Converter and 15 kV SiC MOSFET. In Proceedings of the 2017 IEEE Energy Conversion Congress and Exposition (ECCE), Cincinnati, OH, USA, 1-5 October 2017; pp. 1288-1295.

44. Wang, Z.; Yin, X.; Chen, Y.; Lai, J.; Qi, Z.; Li, L. A Novel Integrated Hybrid Compensation System for Distribution Transformers. In Proceedings of the 2018 International Conference on Power System Technology (POWERCON), Guangzhou, China, 6-8 November 2019; pp. 4424-4430.

45. Lei, E.; Yin, X.; Zhang, Z.; Chen, Y. An Improved Transformer Winding Tap Injection DSTATCOM Topology for Medium-Voltage Reactive Power Compensation. IEEE Trans. Power Electron. 2018, 33, 2113-2126. [CrossRef]

46. Cui, G.; Luo, L.; Li, Y.; Liang, C.; Zhang, X.; Xu, J.; Liu, Y.; Wang, T.; Kubis, A. Active Power Filter Integrated With Distribution Transformer Based on Magnetic Potential Balance. IET Gener. Transm. Distrib. 2019, 13, 238-247. [CrossRef]

47. Wiemer, A.; Biela, J. Comparison of Hybrid Transformers with Uni- and Bidirectional Auxiliary Converter. In Proceedings of the IEEE 2019 21st European Conference on Power Electronics and Applications (EPE '19 ECCE Europe), Genova, Italy, 3-5 September 2019; pp. 1-11.

48. Burkard, J.; Biela, J. Evaluation of Topologies and Optimal Design of a Hybrid Distribution Transformer. In Proceedings of the 2015 17th European Conference on Power Electronics and Applications (EPE'15 ECCE-Europe), Geneva, Switzerland, 8-10 September 2015; pp. 1-10.

49. Kaniewski, J. Hybrid Distribution Transformer Based on a Bipolar Direct AC/AC Converter. IET Electr. Power Appl. 2018, 12, 1034-1039. [CrossRef]

50. Liu, J.; Zeng, H.; Chen, P.; Yang, B.; Wang, J.; Ji, Z.; Song, J. Research on a Novel Hybrid Transformer for Smart Distribution Network. In Proceedings of the 2018 IEEE International Conference on Industrial Technology (ICIT), Lyon, France, 19-22 February 2018; pp. 818-823.

51. Aeloiza, E.; Enjeti, P.; Moran, L.; Pitel, I. Next Generation Distribution Transformer: To Address Power Quality for Critical Loads. In Proceedings of the IEEE 34th Annual Conference on Power Electronics Specialist, 2003. PESC '03, Acapulco, Mexico, 15-19 June 2004; Volume 3, pp. 1266-1271.

52. Ruiz Garcia, O.M.; Gomez Exposito, A. Regulación Continua de la Tensión de Salida en Transformadores Mediante Troceador Reductor. IEEE Lat. Am. Trans. 2007, 5, 137-142.

53. Liu, Y.; Liang, D.; Liang, Y.; Zhang, M.; Chen, Q. Design and Analysis of the Compounded Control System of Hybrid Distribution Transformer. In Proceedings of the 2018 IEEE Energy Conversion Congress and Exposition, ECCE 2018, Portland, OR, USA, 23-27 September 2018; pp. 3664-3668. 
54. Liu, Y.; Liang, D.; Kou, P.; Zhang, M.; Cai, S.; Zhou, K.; Liang, Y.; Chen, Q. Compound Control System of Hybrid Distribution Transformer. IEEE Trans. Ind. Appl. 2020, 56, 6360-6373. [CrossRef]

55. Yu, J.; Xu, Y.; Li, Y.; Liu, Q. An Inductive Hybrid UPQC for Power Quality Management in Premium-Power-Supply-Required Applications. IEEE Access 2020, 8, 113342-113354. [CrossRef]

56. Elsaharty, M.A.; Candela, J.I.; Rodriguez, P. Power System Compensation Using a Power-Electronics Integrated Transformer. IEEE Trans. Power Deliv. 2018, 33, 1744-1754. [CrossRef]

57. Elsaharty, M.A.; Luna, A.; Candela, I.; Rodriguez, P. A Unified Power Flow Controller Using a Power Electronics Integrated Transformer. IEEE Trans. Power Deliv. 2019, 34, 828-839. [CrossRef]

58. Elsaharty, M.A.; Rocabert, J.; Candela, J.I.; Rodriguez, P. Three-Phase Custom Power Active Transformer for Power Flow Control Applications. IEEE Trans. Power Electron. 2019, 34, 2206-2219. [CrossRef]

59. Winter, P.; Cajigal-Nunez, J.M.; Wrede, H.; Schnepp, J. New Topology and Functionalities of a Hybrid Transformer for Flexible Operation of Distribution and Transmission Systems. In Proceedings of the 2019 21st European Conference on Power Electronics and Applications (EPE '19 ECCE Europe), Genova, Italy, 2-5 September 2019; pp. 1-10.

60. Kang, T.; Choi, S.; Morsy, A.S.; Enjeti, P.N. Series Voltage Regulator for a Distribution Transformer to Compensate Voltage Sag/Swell. IEEE Trans. Ind. Electron. 2017, 64, 4501-4510. [CrossRef]

61. Eckhardt, C.; Watts, S.T. Distribution Transformer Interface Apparatus and Methods. US Patent US 10116204 B1, 30 October 2018.

62. Rajendran, S.; Sen, S.; Zhang, L.; Guo, Z.; Huang, Q.; Huang, A.Q. 500 kVA Hybrid Solid State Transformer (HSST): Design and Implementation of the SST. In Proceedings of the 2020 IEEE Energy Conversion Congress and Exposition (ECCE), Detroit, MI, USA, 11-15 October 2020; pp. 1642-1649.

63. Huang, Q.; Rajendran, S.; Sen, S.; Guo, Z.; Zhang, L.; Huang, A.Q. 500 kVA Hybrid Solid State Transformer (HSST): Architecture, Functionality and Control. In Proceedings of the 2020 IEEE Energy Conversion Congress and Exposition (ECCE), Detroit, MI, USA, 11-15 October 2020; pp. 4864-4871.

64. Pinto, S.F.; Alcaria, P.; Monteiro, J.; Silva, J.F. Matrix Converter-Based Active Distribution Transformer. IEEE Trans. Power Deliv. 2016, 31, 1493-1501. [CrossRef]

65. Carreno, A.; Perez, M.; Baier, C.; Espinoza, J. Distribution Network Hybrid Transformer for Load Current and Grid Voltage Compensation. In Proceedings of the IEEE IECON 2019-45th Annual Conference of the IEEE Industrial Electronics Society, Lisbon, Portugal, 14-17 October 2019; Volume 1, pp. 6683-6688.

66. Baier, C.R.; Torres, M.A.; Perez, M.A.; Cárdenas, R.; Ramirez, R.; Melín, P. Hybrid Transformers with Virtual Inertia for Future Distribution Networks. In Proceedings of the IECON 2019-45th Annual Conference of the IEEE Industrial Electronics Society, Lisbon, Portugal, 14-17 October 2019; pp. 6767-6772.

67. Zhu, R.; De Carne, G.; Deng, F.; Liserre, M. Integration of Large Photovoltaic and Wind System by Means of Smart Transformer. IEEE Trans. Ind. Electron. 2017, 64, 8928-8938. [CrossRef]

68. Ramos-Ruiz, J.; Krishnamoorthy, H.; Enjeti, P. Adding Capacity to an Existing Electric Power Distribution Network Using a Solid State Transformer System. In Proceedings of the 2015 IEEE Energy Conversion Congress and Exposition (ECCE), Montreal, QC, Canada, 20-24 September 2015; pp. 6059-6066.

69. Carreno, A.; Perez, M.; Baier, C.; Espinoza, J. Modeling and Control of a Hybrid Transformer based on a Cascaded H-bridge Multilevel Converter. In Proceedings of the IECON 2020 The 46th Annual Conference of the IEEE Industrial Electronics Society, Singapore, 18-21 October 2020; pp. 1614-1619.

70. Das, D.; Kandula, R.P.; Harley, R.; Divan, D.; Schatz, J.; Munoz, J. Design and Testing of a Medium Voltage Controllable Network Transformer Prototype With an Integrated Hybrid Active Filter. In Proceedings of the 2011 IEEE Energy Conversion Congress and Exposition, Phoenix, AZ, USA, 17-22 September 2011; pp. 4035-4042.

71. Das, D.; Kandula, R.P.; Muñoz, J.A.; Divan, D.; Harley, R.G.; Schatz, J.E. An Integrated Controllable Network TransformerHybrid Active Filter System. IEEE Trans. Ind. Appl. 2015, 51, 1692-1701. [CrossRef]

72. Chen, H.; Kandula, R.P.; Prasai, A.; Schatz, J.; Divan, D. Flexible Transformers for Distribution Grid Control. In Proceedings of the 2016 IEEE Energy Conversion Congress and Exposition (ECCE), Milwaukee, WI, USA, 18-22 September 2016; pp. 1-6.

73. Yun, C.G.; Cho, Y. Active Hybrid Solid State Transformer Based on Multi-Level Converter Using SiC MOSFET. Energies 2019, 12, 66. [CrossRef]

74. Van Brunt, E.; Lichtenwalner, D.J.; Leonard, R.; Burk, A.; Sabri, S.; Hull, B.; Allen, S.; Palmour, J.W. Reliability assessment of a large population of $3.3 \mathrm{kV}$, $45 \mathrm{~A} 4 \mathrm{H}-\mathrm{SIC}$ MOSFETs. In Proceedings of the 2017 29th International Symposium on Power Semiconductor Devices and IC's (ISPSD), Sapporo, Japan, 28 May-1 June 2017; pp. 251-254.

75. Sabri, S.; Van Brunt, E.; Barkley, A.; Hull, B.; O'Loughlin, M.; Burk, A.; Allen, S.; Palmour, J. New generation 6.5 kV SiC power MOSFET. In Proceedings of the 2017 IEEE 5th Workshop on Wide Bandgap Power Devices and Applications (WiPDA), Albuquerque, New Mexico, 30 October-1 November 2017; pp. 246-250.

76. Pala, V.; Brunt, E.V.; Cheng, L.; O’Loughlin, M.; Richmond, J.; Burk, A.; Allen, S.T.; Grider, D.; Palmour, J.W.; Scozzie, C.J. 10 kV and $15 \mathrm{kV}$ Silicon Carbide Power MOSFETs for Next-Generation Energy Conversion and Transmission Systems. In Proceedings of the 2014 IEEE Energy Conversion Congress and Exposition (ECCE), Pittsburgh, PA, USA, 14-18 September 2014; pp. 449-454.

77. Sen, S.; Zhang, L.; Zhao, X.; Lei, Y.; Huang, A.Q.; Zhu, Q.; Song, X. Medium Voltage Single-Stage Dual Active Bridge Based Solid State Transformer (DABSST). In Proceedings of the 2018 20th European Conference on Power Electronics and Applications (EPE'18 ECCE Europe), Riga, Latvia, 17-21 September 2018; pp. 1-10. 
78. Rothmund, D.; Guillod, T.; Bortis, D.; Kolar, J.W. 99.1\% Efficient 10 kV SiC-Based Medium-Voltage ZVS Bidirectional Single-Phase PFC AC/DC Stage. IEEE J. Emerg. Sel. Top. Power Electron. 2019, 7, 779-797. [CrossRef]

79. Ansal, V.; Ravikumar, K.; Parthiban, P. Transformerless Dynamic Voltage Restorer for Voltage Sag Mitigation. In Proceedings of the 2016 Biennial International Conference on Power and Energy Systems: Towards Sustainable Energy (PESTSE), Bengaluru, India, 21-23 January 2016.

80. Kouro, S.; Rodriguez, J.; Wu, B.; Bernet, S.; Perez, M. Powering the Future of Industry: High-Power Adjustable Speed Drive Topologies. IEEE Ind. Appl. Mag. 2012, 18, 26-39. [CrossRef]

81. Ertao Lei.; Xianggen Yin.; Yu Chen.; Jian Dai.; Xin Yin. DSTATCOM Connected to the System Through Center-Tapped Distribution Transformer for Reactive Power Compensation. In Proceedings of the 2016 IEEE Power and Energy Society General Meeting (PESGM), Boston, MA, USA, 17-21 July 2016; pp. 1-5.

82. Rosas-Caro, J.C.; Mancilla-David, F.; Gonzalez-Lopez, J.M.; Ramirez-Arredondo, J.M.; Gonzalez-Rodriguez, A.; Salas-Cabrera, N.; Gomez-Garcia, M.; Cisneros-Villegas, H. A Review of AC Choppers. In Proceedings of the 2010 20th International Conference on Electronics Communications and Computers (CONIELECOMP), Cholula, Mexico, 22-24 February 2010; pp. $252-259$.

83. Srinivasan, S.; Venkataramanan, G. Comparative Evaluation of PWM AC-AC Converters. In Proceedings of the PESC '95-Power Electronics Specialist Conference, Atlanta, GA, USA, 18-22 June 1995; Volume 1, pp. 529-535.

84. Kaniewski, J.; Fedyczak, Z.; Benysek, G. AC Voltage Sag/Swell Compensator Based on Three-Phase Hybrid Transformer with Buck-Boost Matrix-Reactance Chopper. IEEE Trans. Ind. Electron. 2014, 61, 3835-3846. [CrossRef]

85. Kaniewski, J.; Fedyczak, Z.; Klytta, M. Implementation of a Three-Phase Hybrid Transformer Using a Matrix Chopper. In Proceedings of the 2009 13th European Conference on Power Electronics and Applications, Barcelona, Spain, 8-10 September 2009; pp. 1-10.

86. Fedyczak, Z.; Kaniewski, J.; Szczesniak, P.; Klytta, M. Modelling and Analysis of Three-Phase Hybrid Transformer With BuckBoost Matrix-Reactance Chopper and Active Load. In Proceedings of the 2014 16th European Conference on Power Electronics and Applications, Lappeenranta, Finland, 26-28 August 2014; pp. 1-10.

87. Fedyczak, Z.; Kaniewski, J.; Klyta, M. Single-Phase Hybrid Transformer Using Matrix-Reactance Chopper With Ćuk Topology. In Proceedings of the 2007 European Conference on Power Electronics and Applications, Aalborg, Denmark, 2-5 September 2007; pp. 1-10.

88. Kaniewski, J.; Jarnut, M.; Szczesniak, P.; Fedyczak, Z. The Study of Smart Distribution Transformer Based on a Bipolar Matrix Chopper. In Proceedings of the 2017 11th IEEE International Conference on Compatibility, Power Electronics and Power Engineering (CPE-POWERENG), Cadiz, Spain, 4-6 April 2017; pp. 282-287.

89. Wang, B. A Series Compensator Based on AC-AC Power Converters With Virtual Quadrature Modulation. In Proceedings of the 2nd International Symposium on Power Electronics for Distributed Generation Systems, Atlanta, GA, USA, 16-20 June 2010; pp. 438-443.

90. Peng, F.Z.; Chen, L.; Zhang, F. Simple Topologies of PWM AC-AC Converters. IEEE Power Electron. Lett. 2003, 1, 10-13. [CrossRef]

91. Deraz, S.A.; Azazi, H.Z.; Zaky, M.S.; Metwaly, M.K.; Dessouki, M.E. Performance Investigation of Three-Phase Three-Switch Direct PWM AC/AC Voltage Converters. IEEE Access 2019, 7, 11485-11501. [CrossRef]

92. Das, D.; Divan, D.M.; Harley, R.G. Power Flow Control in Networks Using Controllable Network Transformers. In Proceedings of the 2009 IEEE Energy Conversion Congress and Exposition, San Jose, CA, USA, 20-24 September 2009; pp. $2224-2231$.

93. Kolar, J.W.; Friedli, T.; Rodriguez, J.; Wheeler, P.W. Review of Three-Phase PWM AC-AC Converter Topologies. IEEE Trans. Ind. Electron. 2011, 58, 4988-5006. [CrossRef]

94. Szczesniak, P.; Kaniewski, J. Hybrid Transformer with Matrix Converter. IEEE Trans. Power Deliv. 2016, 31, 1388-1396. [CrossRef]

95. Perez, M.A.; Rojas, C.A.; Rodriguez, J.; Abu-Rub, H. A Simple Modulation Scheme for a Three-Phase Direct Matrix Converter. In Proceedings of the 2012 IEEE International Symposium on Industrial Electronics, Hangzhou, China, 28-31 May 2012; pp. $105-110$.

96. Strzelecki, R.; Matelski, W.; Tomasov, V. Hybrid Stepless Distribution Transformer With Four-Quadrant AC/DC/AC Converter at Low Voltage Side-Simulation Tests. Prz. Elektrotechniczny 2018, 94, 121-127. [CrossRef]

97. Jinsiwale, R.; Mauger, M.J.; Kandula, R.P.; Divan, D.; Jaroszewski, M.; Schatz, J. Cost-Effective Dynamic Control for Transmission Systems. In Proceedings of the 2018 IEEE Electronic Power Grid (eGrid), Charleston, SC, USA, 12-14 November 2018; pp. 1-6.

98. Qin, H.; Kimball, J.W. Solid-State Transformer Architecture Using AC-AC Dual-Active-Bridge Converter. IEEE Trans. Ind. Electron. 2013, 60, 3720-3730. [CrossRef]

99. Hannan, M.A.; Ker, P.J.; Lipu, M.S.; Choi, Z.H.; Rahman, M.S.A.; Muttaqi, K.M.; Blaabjerg, F. State of the Art of SolidState Transformers: Advanced Topologies, Implementation Issues, Recent Progress and Improvements. IEEE Access 2020, 8, 19113-19132. [CrossRef]

100. Kang, T.; Essakiappan, S.; Enjeti, P.; Choi, S. Towards a Smart Distribution Transformer for Smart Grid. In Proceedings of the 2015 9th International Conference on Power Electronics and ECCE Asia (ICPE-ECCE Asia), Seoul, Korea, 1-5 June 2015; pp. 1997-2003.

101. Strzelecki, R.; Matelski, W.; Malkowski, R.; Tomasov, V.; Wolski, L.; Krahel, A. Distribution Transformer with Multi-Zone Voltage Regulation for Smart Grid System Application. In Proceedings of the 2019 IEEE 6th International Conference on Energy Smart Systems (ESS), Kyiv, Ukraine, 17-19 April 2019; pp. 132-137.

102. Yazdani-Asrami, M.; Mirzaie, M.; Shayegani Akmal, A.A. No-load loss calculation of distribution transformers supplied by nonsinusoidal voltage using three-dimensional finite element analysis. Energy 2013, 50, 205-219. [CrossRef] 
103. Hurley, W.G.; Wölfle, W.H. Transformers and Inductors for Power Electronics; Wiley-Blackwell: Hoboken, NJ, USA, 2013. [CrossRef]

104. Godina, R.; Rodrigues, E.; Matias, J.; Catalão, J. Effect of Loads and Other Key Factors on Oil-Transformer Ageing: Sustainability Benefits and Challenges. Energies 2015, 8, 12147-12186. [CrossRef]

105. Bush, J.; Turk, R.; Myers, K.; Gentle, J.; Baldwin, T. Transformer Efficiency Assessment; Technical Report; INL: Okinawa, Japan, 2012.

106. Ruiz Allende, F.; Perez, M.A.; Espinosa, J.R.; Gajowik, T.; Stynski, S.; Malinowski, M. Surveying Solid-State Transformer Structures and Controls: Providing Highly Efficient and Controllable Power Flow in Distribution Grids. IEEE Ind. Electron. Mag. 2020, 14, 56-70. [CrossRef]

107. Subramaniam, A.; Sahoo, A.; Manohar, S.S.; Panda, S.K. Voltage and current-harmonics induced ageing in electrical insulation. In Proceedings of the 2017 International Symposium on Electrical Insulating Materials (ISEIM), Toyohashi, Japan, 11-15 September 2017; Volume 1, pp. 403-406.

108. Mantilla, H.F.M.; Pavas, A.; Durán, I.C. Aging of distribution transformers due to voltage harmonics. In Proceedings of the 2017 IEEE Workshop on Power Electronics and Power Quality Applications (PEPQA), Bogota, Colombia, 31 May-2 June 2017.

109. El-Bataway, S.A.; Morsi, W.G. Distribution Transformer's Loss of Life Considering Residential Prosumers Owning Solar Shingles, High-Power Fast Chargers and Second-Generation Battery Energy Storage. IEEE Trans. Ind. Inform. 2019, 15, 1287-1297. [CrossRef]

110. Yazdani-Asrami, M.; Mirzaie, M.; Shayegani Akmal, A.A. Investigation on Impact of Current Harmonic Contents on the Distribution Transformer Losses and Remaining Life. In Proceedings of the 2010 IEEE International Conference on Power and Energy, Kuala Lumpur, Malaysia, 29 November-1 December 2010; pp. 689-694.

111. Digalovski, M.; Najdenkoski, K.; Rafajlovski, G. Impact of current high order harmonic to core losses of three-phase distribution transformer. Eurocon 2013, 2013, 1531-1535.

112. Hu, K.; Liu, Z.; Yang, Y.; Iannuzzo, F.; Blaabjerg, F. Ensuring a Reliable Operation of Two-Level IGBT-Based Power Converters: A Review of Monitoring and Fault-Tolerant Approaches. IEEE Access 2020, 8, 89988-90022. [CrossRef]

113. Salimian, H.; Iman-Eini, H. Fault-Tolerant Operation of Three-Phase Cascaded H-Bridge Converters Using an Auxiliary Module. IEEE Trans. Ind. Electron. 2017, 64, 1018-1027. [CrossRef]

114. Oureilidis, K.; Malamaki, K.N.; Gallos, K.; Tsitsimelis, A.; Dikaiakos, C.; Gkavanoudis, S.; Cvetkovic, M.; Mauricio, J.M.; Maza Ortega, J.M.; Ramos, J.L.M.; et al. Ancillary Services Market Design in Distribution Networks: Review and Identification of Barriers. Energies 2020, 13, 917. [CrossRef]

115. Burkard, J.; Biela, J. Transformer Inrush Current Mitigation Concept for Hybrid Transformers. In Proceedings of the IEEE 2017 19th European Conference on Power Electronics and Applications (EPE'17 ECCE Europe), Warsaw, Poland, 11-14 September 2017; Volume 2017, pp. 1-9.

116. Chen, Z.; Li, H.; Liu, L.; Xiang, L.; Bai, B. DC Bias Treatment of Hybrid Type Transformer Based on Magnetic Flux Modulation Mechanism. IEEE Trans. Magn. 2019, 55, 1-4. [CrossRef]

117. Ramos-Ruiz, J.; Morsy, A.; Enjeti, P. Medium Voltage AC-AC Adapter Using Multilevel Capacitor Clamped Buck Converter. In Proceedings of the 2016 13th International Conference on Power Electronics (CIEP), Guanajuato, Mexico, 20-23 June 2016; pp. $35-40$.

118. Fernando, N.; Meegahapola, L.; Thilakarathne, C. Solid State Transformer Parallel Operation With a Tap Changing Line Frequency Transformer. In Proceedings of the 2017 IEEE Innovative Smart Grid Technologies-Asia (ISGT-Asia), Auckland, New Zealand, 27-30 November 2018; pp. 1-6.

119. Shao, H.; Cai, X.; Zhou, D.; Li, Z.; Zheng, D.; Cao, Y.; Wang, Y.; Rao, F. Equivalent Modeling and Comprehensive Evaluation of Inertia Emulation Control Strategy for DFIG Wind Turbine Generator. IEEE Access 2019, 7, 64798-64811. [CrossRef]

120. Kerdphol, T.; Rahman, F.S.; Watanabe, M.; Mitani, Y. Robust Virtual Inertia Control of a Low Inertia Microgrid Considering Frequency Measurement Effects. IEEE Access 2019, 7, 57550-57560. [CrossRef]

121. Kou, P.; Liang, D.; Gao, R.; Liu, Y.; Gao, L. Decentralized Model Predictive Control of Hybrid Distribution Transformers for Voltage Regulation in Active Distribution Networks. IEEE Trans. Sustain. Energy 2019, 11, 2189-2200. [CrossRef] 